Geoffrey Grimmett · Svante Janson

\title{
Branching processes, and random-cluster measures on trees
}

Received October 13, 2004 and in revised form November 3, 2004

\begin{abstract}
Random-cluster measures on infinite regular trees are studied in conjunction with a general type of 'boundary condition', namely an equivalence relation on the set of infinite paths of the tree. The uniqueness and non-uniqueness of random-cluster measures are explored for certain classes of equivalence relations. In proving uniqueness, the following problem concerning branching processes is encountered and answered. Consider bond percolation on the family-tree $T$ of a branching process. What is the probability that every infinite path of $T$, beginning at its root, contains some vertex which is itself the root of an infinite open subtree?
\end{abstract}

Keywords. Branching process, tree, random-cluster measure, mean-field model

\section{Introduction and summary}

The random-cluster model may be viewed as a unification of percolation and the Ising/ Potts models for a ferromagnet. It was described by Fortuin and Kasteleyn around 1970 in a series of influential papers, and has provided one of the principal methods for studying the mathematics of ferromagnetism. See [10, 11] for detailed accounts of and bibliographies associated with the model. When the underlying graph $G$ is finite, the corresponding random-cluster measure is given in a closed form. When the graph is infinite, one proceeds either by taking weak limits of measures on finite subgraphs $H$ as $H \uparrow G$, or by concentrating on a class of measures whose conditional measures, given the configuration off a finite subgraph $H$, satisfy the appropriate 'DLR/Gibbs specification' (see [8]). Much (but not all) is known about the relationship between these two approaches when $G$ is a finite-dimensional lattice. The primary purpose of the present paper is to study the corresponding problem when $G$ is an infinite regular tree, thus continuing a project initiated in [13].

A random-cluster measure on a finite tree is simply a product measure-it is the circuits in a graph which cause dependence between the states of different edges, and, when there are no circuits, there is no dependence. Circuits may, however, be introduced into

G. Grimmett: Statistical Laboratory, Centre for Mathematical Sciences, University of Cambridge, Wilberforce Road, Cambridge CB3 0WB, UK; e-mail: g.r.grimmett@statslab.cam.ac.uk

S. Janson: Department of Mathematics, Uppsala University, PO Box 480, S-751 06 Uppsala, Sweden; e-mail: svante.janson@math.uu.se

Mathematics Subject Classification (2000): 60K35, 60J80, 82B20 
trees through a consideration of boundary conditions, and there lies the principal direction of this paper. Let $T$ be an infinite labelled tree with root 0 , and let $\mathcal{R}$ be the set of all infinite (self-avoiding) paths of $T$ beginning at 0 , termed rays. We may think of a boundary condition as being an equivalence relation $\sim$ on $\mathcal{R}$, the 'physical' meaning being that two rays $\rho, \rho^{\prime}$ are considered to be 'connected at infinity' whenever $\rho \sim \rho^{\prime}$. Such connections affect the counts of connected components of random subgraphs, thereby contributing to the random-cluster measures defined on $T$. The two extremal boundary conditions are usually termed 'free' (meaning that there exist no connections at infinity) and 'wired' (meaning that all rays are equivalent), respectively; these notions agree with those in use for lattices. The wired boundary condition on $T$ is that studied in [13, 18].

Our study of random-cluster measures will be pursued in Sections 4-6 in the context of the infinite $m$-ary tree $T_{m}^{\prime}$, where $m \in\{2,3, \ldots\}$. Let $\sim$ be an equivalence relation on the set $\mathcal{R}$ of rays of $T_{m}^{\prime}$. In Section 4 is presented the DLR/Gibbs specification of a so-called $(\sim)$ random-cluster measure on $T$. When studying random-cluster measures which arise through limits of finite-volume measures, it turns out to be natural to restrict oneself to equivalence classes which are 'closed' when viewed as subsets of $\mathcal{R}^{2}$. Thus we are led to consider the topological properties of equivalence relations, and this we do in Section 5.

A random-cluster measure $\phi_{G, p, q}$ on a graph $G$ has two parameters, namely an edgeweight $p \in[0,1]$ and a cluster-weight $q \in(0, \infty)$. It is an important and useful property of random-cluster measures with $q \geq 1$ that they satisfy the FKG inequality, and for this reason we confine ourselves here to this case. The measure $\phi_{G, p, q}$ increases (in the sense of stochastic ordering) as $p$ increases. When $G$ is infinite, one knows in the case of lattices (see [8]) that there exists a unique random-cluster measure with parameters $p$ and $q(\geq 1)$ whenever $p$ is either sufficiently small or sufficiently large, and it is an important open problem to determine the uniqueness region exactly. The case of small $p$ was answered for $T_{m}^{\prime}$ in [13], where uniqueness was proved for all $p<p_{m, q}$ where $p_{m, q}$ is given by an explicit formula. It was proved moreover that there exists an interval of values of $p$ of the form $\left[p_{m, q}, p_{m, q}^{\prime}\right]$, non-empty when $q>2$, such that there is non-uniqueness of wired random-cluster measures for $p$ lying in this interval. It was conjectured in [13] that uniqueness of wired measures is valid when $p>p_{m, q}^{\prime}$, and such uniqueness was proved in [18] for sufficiently large $p$. In Section 6, we extend the work of [18] to prove the existence of $p_{m, q}^{\prime \prime} \in\left(p_{m, q}^{\prime}, 1\right)$ such that uniqueness is valid for $p \geq p_{m, q}^{\prime \prime}$ in the more general context of a certain subclass of equivalence relations termed 'open' relations.

In proving the above uniqueness, we make use of a result from branching processes which may have other applications also. Let $T$ be the family-tree of a Galton-Watson branching process with a single progenitor 0 , and we assume for simplicity that every family-size is at least 1 and that the mean family-size exceeds 1 . On $T$ we construct a bond percolation process with given edge-density $p$. A vertex $v$ of $T$ is coloured blue if it is the root of an infinite open subtree of $T$. The progenitor 0 is coloured black if every infinite path of $T$ starting at 0 contains some blue vertex. We shall see in Section 2 how to calculate the probability $\gamma=\mathbb{P}(0$ is black $)$ in terms of the family-size probability generating function $G$ of the branching process. 
Of special relevance to our study of random-cluster measures is the problem of finding a necessary and sufficient condition on $p$ such that $\gamma=1$. We shall see in Theorem 2.2 that $\gamma=1$ if and only if $p \geq p_{G}$, where $p_{G}$ is given uniquely by the equation $G^{\prime}\left(1-p_{G} \theta\left(p_{G}\right)\right)=1$, and $\theta(p)$ is the survival probability of the open subtree with root 0 . [We consider here the 'quenched' probability measure which 'averages' over realizations of $T$ as well as over the open edge-set of $T$.] Although we obtain such results in the context of a general branching process, in our application to the random-cluster model, we shall consider (as in [18]) only the deterministic case in which every individual has exactly $m$ children; this is the case with $G(x)=x^{m}$.

The present work is related to the analysis of the random-cluster model on the complete graph performed in [5] and continued in [20], the common concept being that of a 'mean-field model'. A mean-field theory of statistical mechanics arises either through removing the finite-dimensional spatial aspect of the system, or by considering a model which is in some sense 'infinite-dimensional'. In seeking rigorous theory, mathematicians often consider the correct setting for a mean-field model to be either the complete graph or a tree. In the case of percolation, for example, the corresponding models are the socalled Erdős-Rényi random graph (see [4, 17]) and the binomial branching process (see [9. Chapter 10]). Paper [5] contains the theory of the random-cluster measure on the complete graph with $n$ vertices, where $q \in(0, \infty)$ and $p=\lambda / n$, in the limit as $n \rightarrow \infty$. The present paper (and the earlier [13, 18]) is devoted to the case of trees.

\section{Branching processes}

We pose and answer a natural question concerning branching processes. This has an application for the uniqueness of random-cluster measures on trees, and it may well have further applications in other areas of probability theory. It may be viewed as an extension of a (sub-)result of [18].

We consider a (Galton-Watson) branching process with family-size probability generating function $G$ satisfying

$$
G(0)=0, \quad 1<G^{\prime}(1)<\infty .
$$

In other words, the number of offspring of any individual is non-zero, and the mean family-size is strictly greater than 1 (we shall return after Corollary 2.3 to the situation in which one dispenses with the assumption $G(0)=0$ ). The family-tree $T$ of the process is an infinite tree with labelled vertex-set $V$ and a single progenitor called its origin and labelled 0 . We write $\mathbb{P}$ for the probability measure which governs the branching process. For general accounts of the theory of branching processes, see [1, 15, 16].

We turn $T$ into a directed tree by directing every edge away from 0 . Let $x$ be a vertex. An $x$-ray is defined to be an infinite directed path of $T$ with (unique) endvertex $x$. We denote by $\mathcal{R}_{x}$ the set of all $x$-rays of $T$, and we abbreviate $\mathcal{R}_{0}$ to $\mathcal{R}$. We shall use the term ray to mean a member of some $\mathcal{R}_{x}$. The edge of $T$ joining vertices $x$ and $y$ is denoted $\langle x, y\rangle$ when undirected, and $[x, y\rangle$ when directed from $x$ to $y$. 
We introduce next a second level of randomness through a consideration of bond percolation on $T$ (see [9] for a general account of percolation). Suppose for the moment that $T$ is given, which is to say that $T=(V, E)$ is a given labelled directed rooted infinite tree as above. Let $0 \leq p \leq 1$. Each edge of $T$ is declared open with probability $p$, and closed otherwise; the states of different edges are independent. This amounts to considering the product measure $P_{E, p}$ with density $p$ on the configuration space $\{0,1\}^{E}$. Let $\widetilde{E}(\subseteq E)$ be the set of open edges, and define the forest $\widetilde{T}=(V, \widetilde{E})$. It is standard that the connected component of $\widetilde{T}$ containing 0 is itself a branching process. A path $\pi$ of $T$ is called open if every edge in $\pi$ is open.

The vertices of $T$ are assigned colours depending on the subtrees of which they are roots. Let $x \in V$. We colour $x$ blue if some $x$-ray of $T$ is open; we colour $x$ yellow if $x$ is not blue but every $x$-ray of $T$ contains some blue vertex; we colour $x$ red if it is neither blue nor yellow. Finally, a vertex which is either blue or yellow is said to be black. Note that $x$ is black if and only if every $x$-ray contains a blue vertex. We write

$$
\gamma_{T, x}=P_{E, p}(x \text { is black }), \quad \theta_{T, x}=P_{E, p}(x \text { is blue }),
$$

noting that these quantities depend on the tree $T$. We now average over the measure $\mathbb{P}$. Let $k \geq 0$ and let $\mathcal{F}_{k}$ be the $\sigma$-field generated by the first $k$ generations of $T$. Suppose that $v$ lies in the $k$ th generation of $T$. By the Markov property of branching processes, the quantities

$$
\gamma_{v}=\mathbb{P}\left(\gamma_{T, v} \mid \mathcal{F}_{k}\right), \quad \theta_{v}=\mathbb{P}\left(\theta_{T, v} \mid \mathcal{F}_{k}\right)
$$

do not depend (almost surely) on the choice of $v$ and $k$, whence

$$
\gamma_{v}=\gamma_{0}, \quad \theta_{v}=\theta_{0} .
$$

[Rather than introduce further notation, we use $\mu(X)$ to denote the mean of a random variable $X$ under a probability measure $\mu$.] We introduce the abbreviations

$$
\gamma=\gamma_{0}, \quad \theta=\theta_{0},
$$

and we note the obvious inequality

$$
\gamma \geq \theta
$$

In summary, the root 0 is blue (respectively, red, yellow) with probability $\theta$ (respectively, $1-\gamma, \gamma-\theta)$; it is black with probability $\gamma$.

The calculation of $\theta=\theta(p, G)$ is standard, and may be found in any of many textbooks (see, for example, [12] Thm 5.4.5]). The extinction probability $\eta=1-\theta$ is the smallest positive root of the equation

$$
\eta=G(1-p+p \eta),
$$

and thus $\theta$ is the largest root in $[0,1]$ of the equation

$$
\theta=1-G(1-p \theta)
$$

It follows from (2.7) in the usual manner that

$$
\theta>0 \text { if and only if } p G^{\prime}(1)>1 .
$$



by

Our principal target in this section is to calculate $\gamma$. We define $f_{p}:[p \theta, 1] \rightarrow[0, \infty)$

$$
f_{p}(\alpha)=\theta+G(\alpha-p \theta), \quad \alpha \in[p \theta, 1],
$$

and we note some properties of $f_{p}$ the proofs of which will be given later.

Proposition 2.1. Let $p \in[0,1)$ and let $G$ satisfy $(2.1)$ The equation $\alpha=f_{p}(\alpha)$ has a root at $\alpha=1$. It has either one or two roots in the interval $[p \theta, 1]$, and it has two distinct roots in this interval if and only if $G^{\prime}(1-p \theta)>1$.

The function $f_{p}$ is sketched in Figure 2.1. Next is the main result of this section.

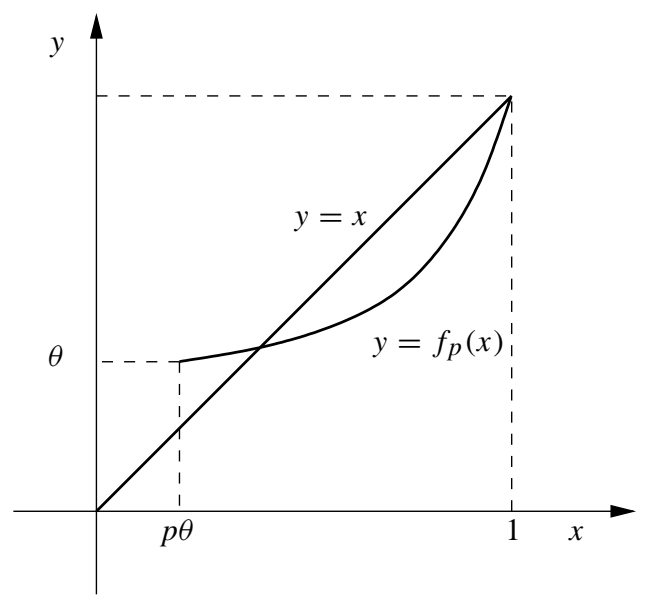

Fig. 2.1. A sketch of the graphs of $y=x$ and $y=f_{p}(x)$ on the interval $[p \theta, 1]$. Whether or not there exists a root of $\alpha=f_{p}(\alpha)$ other than at $\alpha=1$ depends on the gradient of $f_{p}$ at $\alpha=1$.

Theorem 2.2. Consider a branching process whose family-size probability generating function satisfies (2.1)] and let $p \in[0,1)$. Then $\gamma$ is the smallest root in the interval $[p \theta, 1]$ of the equation

$$
\gamma=\theta+G(\gamma-p \theta)
$$

The following are equivalent:

(i) $\gamma=1$, which is to say there exist almost surely no red vertices,

(ii) $G^{\prime}(1-p \theta) \leq 1$,

(iii) $p_{G} \leq p \leq 1$, where $p_{G}$ is given uniquely by $G^{\prime}\left(1-p_{G} \theta\left(p_{G}\right)\right)=1$.

We make a remark about the value $p_{G}$ given in Theorem 2.2(iii). The function $\theta=\theta(p)$ is smooth when $p>p_{0}=1 / G^{\prime}(1)$. On differentiating (2.8) we find that

$$
\theta^{\prime}=\left(\theta+p \theta^{\prime}\right) G^{\prime}(1-p \theta) .
$$

Hence, for $p_{0}<p<1, G^{\prime}(1-p \theta) \leq 1$ if and only if $\theta^{\prime} \leq \theta+p \theta^{\prime}$, which is to say that $\frac{d}{d p}((1-p) \theta(p))=(1-p) \theta^{\prime}-\theta \leq 0$.

Therefore, $p_{G}$ is characterised as the value of $p \in[0,1]$ which maximises $(1-p) \theta(p)$. 
We point out that the coloured tree $T$ constitutes a multi-type branching process. That is, suppose that each vertex of $T$ is coloured red, blue, or yellow in the manner described above. We may think of the colour of any given vertex as its type, and then it is an exercise in the theory of multi-type branching processes to show that $T$ (when coloured) has the same distribution as the family-tree of a multi-type branching process with certain offspring-type distributions. This is a consequence of a general result for multi-type processes which may already be known, and whose details are contained in the next section.

We turn briefly to a particular instance of importance for the random-cluster model on a regular tree. Let $m \in\{2,3, \ldots\}$ be a given integer, and let $T_{m}$ denote the infinite labelled rooted tree in which the root has degree $m$ and every other vertex has degree $m+1$. This is the family-tree of the branching process with probability generating function given by $G(\alpha)=\alpha^{m}, \alpha \in \mathbb{R}$. We consider bond percolation on $T_{m}$ with edge-density $p$ as above, and we arrive at the following result to be found in [18].

Corollary 2.3 ([18]). Let $m \in\{2,3, \ldots\}$ and $p \in[0,1]$. The probability $\gamma$ that the root of $T_{m}$ is black satisfies $\gamma=1$ if and only if $p \geq p_{\mathrm{b}}(m)$, where

$$
p_{\mathrm{b}}(m)=\frac{1-m^{-1 /(m-1)}}{1-m^{-m /(m-1)}} .
$$

In particular,

$$
p_{\mathrm{b}}(2)=\frac{2}{3}, \quad p_{\mathrm{b}}(3)=\frac{3}{13}(4-\sqrt{3}) \approx 0.52337 \ldots,
$$

and it is easily seen that

$$
p_{\mathrm{b}}(m) \sim \frac{\log m}{m} \quad \text { as } m \rightarrow \infty .
$$

Finally, prior to the proofs, we make a remark about the situation when (2.1) is not assumed in its entirety, but only that $1<G^{\prime}(1)<\infty$. The branching process is then supercritical, but may be finite with a strictly positive probability. Even if $T$ is infinite, it will generally contain vertices $x$ for which the set $\mathcal{R}_{x}$ of $x$-rays is empty, and such vertices are automatically assigned the colour yellow, following the rules given towards the start of this section. The conclusion of Theorem 2.2 is easily seen to be valid in this more general setting.

Proof of Proposition 2.1. The function $f_{p}$ is non-decreasing and strictly convex on the interval $[p \theta, 1]$. It is clear by $(2.8)$ that $f_{p}(1)=1$ and $f_{p}(p \theta) \geq p \theta$. See Figure 2.1.

If $f_{p}^{\prime}(1)>1$, there exist two distinct roots of the equation $\alpha=f_{p}(\alpha)$ in $[p \theta, 1]$; if $f_{p}^{\prime}(1) \leq 1$, then $\alpha=1$ is the unique such root.

Proof of Theorem 2.2. Let $k \geq 1$, and let $X$ be the number of offspring of the progenitor 0 . We say that a vertex $x$ is $k$-yellow if: $x$ is not blue, but every $x$-ray contains a blue vertex belonging to the first $k$ generations of $T$. Vertex $x$ is called $k$-black if it is either blue or $k$-yellow. Let $\gamma(k, X)$ be the (conditional) probability given $X$ that 0 is $k$-black, and write $\gamma(k)=\mathbb{P}(\gamma(k, X))$. Now, 0 is $k$-yellow if and only if it is not blue but every 
child is either $(k-1)$-yellow or blue. This occurs if and only if every child $y$ of 0 satisfies: either $y$ is $(k-1)$-yellow, or $y$ is blue and the edge $\langle 0, y\rangle$ is closed. Therefore,

$$
[\gamma(k, X)-\theta]=([\gamma(k-1)-\theta]+(1-p) \theta)^{X} .
$$

We take expectations to find that

$$
\gamma(k)=f_{p}(\gamma(k-1))
$$

where $f_{p}$ is given in (2.10). Now $\gamma(k) \rightarrow \gamma$ as $k \rightarrow \infty$, and $f_{p}$ is continuous, whence $\gamma$ satisfies (2.11) Since $\gamma(0)=\theta<1$, we find by the usual recursion argument that $\gamma$ is the smallest root in $[p \theta, 1]$ of (2.11) Note that $\gamma \geq \theta$ by (2.6)

By Proposition 2.1. $\gamma=1$ if and only if $f_{p}^{\prime}(1)=G^{\prime}(1-p \theta) \leq 1$. Now $G$ is strictly convex and differentiable on $[0,1]$, and $G^{\prime}(1)>1$ by $(2.1)$, while $G^{\prime}(0)=\mathbb{P}(X=1)<1$. Therefore there exists a unique $\beta \in(0,1)$ such that $G^{\prime}(\beta)=1$, and $G^{\prime}(1-p \theta) \leq 1$ if and only if $1-p \theta \leq \beta$.

Proof of Corollary 2.3. Since $G(\alpha)=\alpha^{m}$, the unique root of the equation $G^{\prime}(\beta)=1$ is given by $\beta=m^{-1 /(m-1)}$. If $p \theta=1-\beta$ then, by (2.8).

$$
1-\theta=G(\beta)=\beta^{m}
$$

whence

as claimed.

$$
p=\frac{1-\beta}{\theta}=\frac{1-m^{-1 /(m-1)}}{1-m^{-m /(m-1)}}
$$

\section{Multi-type branching processes}

We prove a general result about multi-type processes in this section, and then apply it to the coloured branching processes of Section 2. A related argument underpins the Markovian construction of random-cluster measures on trees in [13].

Consider a multi-type (Galton-Watson) branching process with a set $\mathcal{I}$ of types; $\mathcal{I}$ may be finite or countably infinite. We assume, for convenience, that the children of each individual are ordered in some manner, and we may if necessary impose a random ordering within families. Suppose also that we are given a (measurable) classification of the possible family-trees into a (finite or countable) set of types $\mathcal{J}$ that we call 'colours' (not to be confused with the original types that will be called 'types'). We colour each vertex $x$ of the family-tree by the colour of the subtree rooted at $x$.

We shall assume that the colouring rule has the property that the colour of any given vertex is determined by the number and types and colours of its children. If $x$ has $k$ offspring labelled $1,2, \ldots, k$, the $r$ th of which has type $i_{r}$ and colour $j_{r}$, we denote the colour of $x$ by $\Phi\left(k ; i_{1}, j_{1}, \ldots, i_{k}, j_{k}\right)$.

Theorem 3.1. The coloured family-tree of the process, with vertices marked by both type and colour, is a multi-type branching process with type space $\mathcal{I} \times \mathcal{J}$. 
Proof. Let $p_{i}\left(k ; i_{1}, \ldots, i_{k}\right)$ be the probability that an individual of type $i \in \mathcal{I}$ has $k \geq 0$ children of types $i_{1}, \ldots, i_{k}$, respectively. Let $q_{i j}$ be the probability that the family-tree, starting with an individual of type $i \in \mathcal{I}$, receives colour $j \in \mathcal{J}$.

Let

$$
\begin{aligned}
p_{i j}\left(k ; i_{1}, j_{1}, \ldots, i_{k}, j_{k}\right) & \\
& = \begin{cases}q_{i j}^{-1} p_{i}\left(k ; i_{1}, \ldots, i_{k}\right) \prod_{l=1}^{k} q_{i_{l} j_{l}} & \text { if } \Phi\left(k ; i_{1}, j_{1}, \ldots, i_{k}, j_{k}\right)=j, \\
0 & \text { otherwise. }\end{cases}
\end{aligned}
$$

There is probability $p_{i}\left(k ; i_{1}, \ldots, i_{k}\right) \prod_{l=1}^{k} q_{i_{l} j_{l}}$ that a family-tree starting with an individual of type $i$ has $k$ children with types and colours $\left(i_{1}, j_{1}\right), \ldots,\left(i_{k}, j_{k}\right)$. In this case the root is coloured $\Phi\left(k ; i_{1}, j_{1}, \ldots, i_{k}, j_{k}\right)$, and thus the probability of this happening and the root being coloured $j$ is $q_{i j} p_{i j}\left(k ; i_{1}, j_{1}, \ldots, i_{k}, j_{k}\right)$. Consequently, the conditional probability that this happens given that the root has type $i$ and colour $j$ is $p_{i j}\left(k ; i_{1}, j_{1}, \ldots, i_{k}, j_{k}\right)$.

Moreover, if we label the $k$ children of the root as $x_{1}, \ldots, x_{k}$, and we require some further events $\mathcal{E}_{1}, \ldots, \mathcal{E}_{k}$, where $\mathcal{E}_{l}$ depends only on the subtree rooted at $x_{l}$, then in these probabilities we have to replace $q_{i_{l} j_{l}}$ by

$\mathbb{P}\left(\mathcal{E}_{l}\right.$, and $x_{l}$ receives colour $j_{l} \mid x_{l}$ has type $\left.i_{l}\right)$

$$
=q_{i_{l} j_{l}} \mathbb{P}\left(\mathcal{E}_{l} \mid x_{l} \text { has type } i_{l} \text { and colour } j_{l}\right) .
$$

Thus the probabilities are multiplied by

$$
\prod_{l=1}^{k} \mathbb{P}\left(\mathcal{E}_{l} \mid x_{l} \text { has type } i_{l} \text { and colour } j_{l}\right) .
$$

Consequently, given the types and colours in the first generation, the $k$ branches are independent of each other and are copies of the entire coloured family-tree, with appropriate initial conditions. In other words, the coloured family-tree is a multi-type branching process with type space $\mathcal{I} \times \mathcal{J}$, where the probability that a particle of type and colour $(i, j) \in \mathcal{I} \times \mathcal{J}$ has $k \geq 0$ children of types and colours $\left(i_{1}, j_{1}\right), \ldots,\left(i_{k}, j_{k}\right)$, respectively, is $p_{i j}\left(k ; i_{1}, j_{1}, \ldots, i_{k}, j_{k}\right)$.

Let us apply this result to the coloured trees of Section 2 . The tree $T$ is the familytree of a branching process with probability generating function $G$ satisfying (2.1) We designate any given edge of $T$ as 'open' with probability $p$, and as 'closed' otherwise. Thus the 'type' of any vertex of $T$ is taken to be the state of the incoming edge, namely either 1 (open) or 0 (closed). (The type of the root of the tree is irrelevant.) The number of offspring of $x$ thus does not depend on the type of $x$, and each child is assigned a type independently of the types of the other offspring, with probability $\pi_{0}=1-p$ (respectively, $\pi_{1}=p$ ) for type 0 (respectively, type 1). We may thus write $q_{j}$ for the probability that the root receives colour $j \in\{b, y, r\}$. 
Consider for example the binary tree $T_{2}$ defined at the end of Section 2 . We have

$$
\begin{aligned}
& p_{b}\left(i_{1}, j_{1}, i_{2}, j_{2}\right)= \begin{cases}p \pi_{i_{2}} q_{j_{2}} & \text { if }\left(i_{1}, j_{1}\right)=(1, b), \\
p \pi_{i_{1}} q_{j_{1}} & \text { if }\left(i_{2}, j_{2}\right)=(1, b), \\
0 & \text { otherwise; }\end{cases} \\
& p_{y}\left(i_{1}, j_{1}, i_{2}, j_{2}\right)= \begin{cases}\pi_{i_{1}} \pi_{i_{2}} q_{j_{2}} & \text { if } j_{1}=y \text { and either } j_{2}=y \text { or }\left(i_{2}, j_{2}\right)=(0, b), \\
\pi_{0} \pi_{i_{2}} q_{b} & \text { if }\left(i_{1}, j_{1}\right)=(0, b) \text { and } j_{2}=y, \\
\pi_{0}^{2} q_{b}^{2} / q_{y} & \text { if }\left(i_{1}, j_{1}\right)=\left(i_{2}, j_{2}\right)=(0, b), \\
0 & \text { otherwise; }\end{cases} \\
& p_{r}\left(i_{1}, j_{1}, i_{2}, j_{2}\right)= \begin{cases}\pi_{i_{1}} \pi_{i_{2}} q_{j_{2}} & \text { if } j_{1}=r \text { and }\left(i_{2}, j_{2}\right) \neq(1, b), \\
\pi_{i_{1}} \pi_{i_{2}} q_{j_{1}} & \text { if } j_{2}=r \text { and }\left(i_{1}, j_{1}\right) \neq(1, b), \\
0 & \text { otherwise. }\end{cases}
\end{aligned}
$$

The following is easily seen. The mean number of red offspring of a red individual is

$$
\mu_{r}=2\left(1-p q_{b}\right)=2(1-p \theta)
$$

and $\mu_{r}>1$ if and only if $p<p_{\mathrm{b}}(2)=\frac{2}{3}$; see (2.12) and Theorem 2.2. Therefore, the red subtree of the multi-type process having red progenitor is supercritical if and only if $p<p_{\mathrm{b}}(2)$. A similar calculation is valid for $T_{m}$ with $m \geq 2$.

In fact, for any branching process as in Section 2, a simple calculation shows that the mean number of red offspring of a red individual is $G^{\prime}(1-p \theta)$.

\section{Random-cluster measures on trees}

Henceforth, we restrict ourselves to the regular infinite labelled $m$-ary tree $T_{m}^{\prime}=(V, E)$, where $m \in\{2,3, \ldots\}$. Each vertex has degree $m+1$, and there is a distinguished origin labelled 0 . We shall state our results for general $m$, but may sometimes consider the special case $m=2$ for simplicity. Part of $T_{2}^{\prime}$ is drawn in Figure 4.1 .

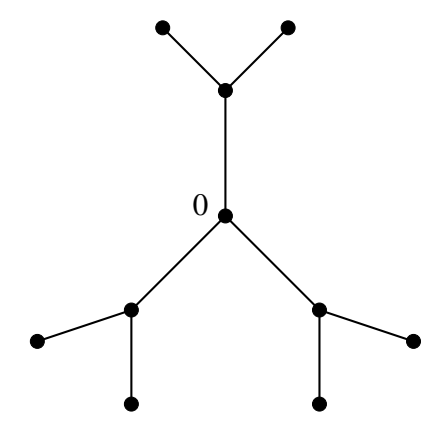

Fig. 4.1. Part of the infinite binary tree $T_{2}^{\prime}$. 
The tree $T_{m}^{\prime}$ differs from $T_{m}$ only in the degree of its root. We have chosen to work with $T_{m}^{\prime}$ rather than $T_{m}$ in this section only because this is the more natural setting for the random-cluster model. Since $T_{m}^{\prime}$ is a regular tree, it has a larger family of graphautomorphisms. When it comes to calculations of critical values and the like, the differences between $T_{m}^{\prime}$ and $T_{m}$ are largely cosmetic.

We continue the study of random-cluster measures on $T_{m}^{\prime}$ initiated in [13], beginning with a more general definition than that used there. Let $\Omega=\{0,1\}^{E}$, and equip $\Omega$ with the $\sigma$-field $\mathcal{F}$ generated by the finite-dimensional cylinder sets. An edge $e$ is called open in a configuration $\omega(\in \Omega)$ if $\omega(e)=1$, and closed otherwise. We write $\eta(\omega)=\{e$ : $\omega(e)=1\}$ for the set of open edges in $\omega$. We shall consider probability measures on the measurable pair $(\Omega, \mathcal{F})$ which satisfy a certain 'random-cluster' condition. Since $T_{m}^{\prime}$ contains no circuits, random-cluster measures on $T_{m}^{\prime}$ are simply product measures. A much more interesting structure is revealed through the introduction of the concept of 'boundary conditions'. A similar development for Ising models has been explored in the statistical physics literature (see [2, 3]), and in the probability literature under the title 'broadcasting in trees' (see [21, 22]). Boundary conditions may be introduced in the more general context of non-amenable graphs, but we do not follow this route here; see [11, 14, 18] for accounts of the random-cluster model on a non-amenable graph.

Each edge of $T_{m}^{\prime}$ is directed away from the root 0 . We shall make use of the rays of $T_{m}^{\prime}$ and we remind the reader of the notation concerning rays at the beginning of Section 2. The set $\mathcal{R}$ of 0 -rays of $T_{m}^{\prime}$ is in one-one correspondence with the set $\{1,2, \ldots, m+1\} \times$ $\{1,2, \ldots, m\}^{\mathbb{N}}$. Let $\sim$ be an equivalence relation on $\mathcal{R}$, and write $\mathcal{C}(\sim)$ for the family of equivalence classes of $\sim$. Let $\mathcal{E}$ denote the class of all equivalence relations on $\mathcal{R}$. There is a partial order $\leq$ on $\mathcal{E}$ given by: $\sim_{1} \leq \sim_{2}$ if

$$
\text { for all } \rho, \rho^{\prime} \in \mathcal{R}, \rho \sim_{2} \rho^{\prime} \text { whenever } \rho \sim_{1} \rho^{\prime} .
$$

There is a minimal (respectively, maximal) equivalence relation in this partial order which we denote as $\sim^{0}$ (respectively, $\sim^{1}$ ). The equivalence classes of $\sim^{0}$ are singletons, whereas $\mathcal{C}\left(\sim^{1}\right)=\{\mathcal{R}\}$.

For $x \in V$, let $\Pi_{x}$ be the set of infinite (undirected) paths of $T_{m}^{\prime}$ with endpoint $x$. Let $x \in V$ and let $\pi \in \Pi_{x}$; there exists a unique 0 -ray, denoted $\rho_{\pi}$, such that $\pi$ and $\rho_{\pi}$ differ on only finitely many edges. For given $x$, this gives a one-one correspondence $\pi \leftrightarrow \rho_{\pi}$ between $\Pi_{x}$ and $\mathcal{R}$. (For $x=0$, it is the identity.) Any relation $\sim$ on $\mathcal{R}$ may be extended to a relation on $\bigcup_{v \in V} \Pi_{v}$ by: for $\pi \in \Pi_{u}, \tau \in \Pi_{v}$, we have $\pi \sim \tau$ if and only if $\rho_{\pi} \sim \rho_{\tau}$.

For any vertex $u \in V$, we write $\mathcal{R}_{u}^{\prime}$ for the subset of $\mathcal{R}$ comprising all rays which pass through $u$. The correspondence between $\Pi_{u}$ and $\mathcal{R}$ restricts to a correspondence between their subsets $\mathcal{R}_{u}$ and $\mathcal{R}_{u}^{\prime}$, such that any ray $\rho_{u} \in \mathcal{R}_{u}$ corresponds to the unique ray $\rho_{u}^{\prime} \in \mathcal{R}$ of which it is a subray.

The equivalence relation $\sim(\in \mathcal{E})$ may serve as a boundary condition, to be interpreted roughly as follows. Suppose that $\omega \in \Omega$, and let $C_{1}, C_{2}$ be two distinct connected components of the graph $(V, \eta(\omega))$. Then $C_{1}$ and $C_{2}$ are considered to be the same cluster if there exist rays $\rho_{1} \subseteq C_{1}, \rho_{2} \subseteq C_{2}$ such that $\rho_{1} \sim \rho_{2}$. Otherwise expressed, two rays are 'identified at infinity' if they are equivalent under $\sim$. This will be made more rigorous in the following formal definition of a random-cluster measure. 
Let $\Lambda$ be a finite subset of $V$, and let $E_{\Lambda}$ be the set of edges of $T_{m}^{\prime}$ having both endvertices in $\Lambda$. For $\xi \in \Omega$, we write $\Omega_{\Lambda}^{\xi}$ for the (finite) subset of $\Omega$ containing all configurations $\omega$ satisfying $\omega(e)=\xi(e)$ for $e \in E \backslash E_{\Lambda}$; these are the configurations which 'agree with $\xi$ off $\Lambda$ '.

Let $\sim \in \mathcal{E}, \xi \in \Omega$, and $\omega \in \Omega_{\Lambda}^{\xi}$. The configuration $\omega$ gives rise to an 'open graph' on $\Lambda$, namely $G(\Lambda, \omega)=\left(\Lambda, \eta(\omega) \cap E_{\Lambda}\right)$. We augment this graph by adding certain new edges. Specifically, for distinct $u, v \in \Lambda$, we add a new edge between the pair $u, v$ if either:

(a) there exists a path of $E \backslash E_{\Lambda}$ from $u$ to $v$ which is open in $\xi$, or

(b) there exists a vertex-disjoint pair of infinite paths $\pi_{u} \in \Pi_{u}, \pi_{v} \in \Pi_{v}$ satisfying $\pi_{u} \sim \pi_{v}$, which are open in $\xi$ and which are edge-disjoint from $E_{\Lambda}$.

We write $G^{\xi, \sim}(\Lambda, \omega)$ for the resulting augmented graph, and we let $k^{\xi, \sim}(\Lambda, \omega)$ be the number of connected components of $G^{\xi, \sim}(\Lambda, \omega)$. These definitions are motivated by the idea that each equivalence class of rays leads to a common 'point at infinity', through which vertices $u$ and $v$ may be connected by open paths.

Next we define a random-cluster measure corresponding to a given equivalence relation $\sim$. Let $\sim \in \mathcal{E}, \xi \in \Omega$, and let $p \in[0,1]$ and $q \in(0, \infty)$. We define $\phi_{\Lambda, p, q}^{\xi, \sim}$ to be the random-cluster measure on the finite graph $\left(\Lambda, E_{\Lambda}\right)$ 'with boundary condition $\xi$ '. More precisely, let $\phi_{\Lambda, p, q}^{\xi, \sim}$ be the probability measure on the pair $(\Omega, \mathcal{F})$ given by

$$
\phi_{\Lambda, p, q}^{\xi, \sim}(\omega)= \begin{cases}\frac{1}{Z_{\Lambda, p, q}^{\xi, \sim}}\left\{\prod_{e \in E_{\Lambda}} p^{\omega(e)}(1-p)^{1-\omega(e)}\right\} q^{k^{\xi, \sim}(\Lambda, \omega)} & \text { if } \omega \in \Omega_{\Lambda}^{\xi} \\ 0 & \text { otherwise }\end{cases}
$$

where $Z_{\Lambda, p, q}^{\xi, \sim}$ is the appropriate normalising constant

$$
Z_{\Lambda, p, q}^{\xi, \sim}=\sum_{\omega \in \Omega_{\Lambda}^{\xi}}\left\{\prod_{e \in E_{\Lambda}} p^{\omega(e)}(1-p)^{1-\omega(e)}\right\} q^{k^{\xi, \sim}(\Lambda, \omega)} .
$$

Note that $\phi_{\Lambda, p, q}^{\xi, \sim}\left(\Omega_{\Lambda}^{\xi}\right)=1$.

In the special case when $\xi=1$ and $\sim=\sim 1$, we write $\phi_{\Lambda, p, q}^{1}$ for $\phi_{\Lambda, p, q}^{\xi, \sim}$. This is usually called the random-cluster measure on $\Lambda$ with 'wired' boundary conditions, and it has been studied in a slightly disguised form in [13]. As usual (see [10] and the references therein), random-cluster measures satisfy the FKG inequality when $q \geq 1$, and this allows the deduction that the weak limit

$$
\phi_{p, q}^{1}=\lim _{\Lambda \uparrow V} \phi_{\Lambda, p, q}^{1}
$$

exists when $q \geq 1$, and is independent of the manner in which the limit $\Lambda \uparrow V$ is taken. As a side-comment, we remark that the FKG inequality is a fundamental technique in the study of the random-cluster model. This is already very familiar in the field (see [10], for example), and we do not explain it further here. Thus, we shall omit details 
of arguments involving the FKG inequality and the stochastic ordering relation $\leq_{\mathrm{st}}$ of probability measures. We note for later use that, for all $q \geq 1$ and $\sim \in \mathcal{E}$,

$$
\phi_{\Delta, p, q}^{1, \sim} \leq_{\mathrm{st}} \phi_{\Lambda, p, q}^{1, \sim} \quad \text { if } \Delta \supseteq \Lambda .
$$

For any finite subset $\Lambda \subseteq V$, we write $\mathcal{T}_{\Lambda}$ for the $\sigma$-field generated by the set $\{\omega(e)$ : $\left.e \in E \backslash E_{\Lambda}\right\}$ of states of edges having at least one endvertex outside $\Lambda$.

Let $\sim \in \mathcal{E}, 0 \leq p \leq 1$, and $q>0$. A probability measure $\phi$ on $(\Omega, \mathcal{F})$ is called a $(\sim)$ random-cluster measure with parameters $p$ and $q$ if: for all $A \in \mathcal{F}$ and all finite subsets $\Lambda \subseteq V$,

$$
\phi\left(A \mid \mathcal{T}_{\Lambda}\right)(\xi)=\phi_{\Lambda, p, q}^{\xi, \sim}(A) \quad \text { for } \phi \text {-a.e. } \xi .
$$

The set of such measures is denoted $\mathcal{R}_{p, q}^{\sim}$. Random-cluster measures were introduced in [8] and are studied systematically in [11] and elsewhere. Note that the cases when $p=0$, $p=1$ or $q=1$ are trivial; in these cases, (4.2) gives the product measure on $\left(\Lambda, E_{\Lambda}\right)$ for every $\xi$ and $\sim$, and then (4.6) holds if and only if $\phi$ is the product measure $\phi_{p}$ with density $p$ on $(\Omega, \mathcal{F})$.

This is an appropriate moment to introduce a measurability assumption on $\sim$. The left hand side of (4.6) is a measurable function of $\xi$, so we want the right hand side to be a measurable function of $\xi$ too, with respect to $\mathcal{F}$ or at least with respect to its completion for $\phi$. For $\Lambda$ a finite subset of $V$ and $u, v \in \Lambda$, let $K_{u, v, \Lambda}^{\sim}$ denote the set of $\omega \in \Omega=$ $\{0,1\}^{E}$ such that there exist infinite vertex-disjoint paths $\pi_{u} \in \Pi_{u}, \pi_{v} \in \Pi_{v}$ satisfying $\pi_{u} \sim \pi_{v}$ and that are open in $\omega$ and edge-disjoint from $E_{\Lambda}$. We call the equivalence relation $\sim$ measurable if $K_{u, v, \Lambda}^{\sim} \in \mathcal{F}$ for all such $u, v, \Lambda$. It is an easy exercise to deduce, if $\sim$ is measurable, that $\phi_{\Lambda, p, q}^{\xi, \sim}(A)$ is a measurable function of $\xi$, thus permitting condition $(4.6)$. We write $\mathcal{E}_{\mathrm{m}}$ for the set of all measurable members of $\mathcal{E}$. We discuss measurability further in the next section.

Returning to the extremal equivalence relations $\sim^{0}, \sim^{1}$, for simplicity of notation we write $\mathcal{R}_{p, q}^{\sim 0}=\mathcal{R}_{p, q}^{0}$ and similarly $\mathcal{R}_{p, q}^{\sim 1}=\mathcal{R}_{p, q}^{1}$. Members of $\mathcal{R}_{p, q}^{0}$ (respectively, $\mathcal{R}_{p, q}^{1}$ ) are called 'free' random-cluster measures (respectively, 'wired' random-cluster measures).

The basic questions of interest include the following. For what $\sim \in \mathcal{E}_{\mathrm{m}}, p, q$ is the set $\mathcal{R}_{p, q}^{\sim}$ non-empty, and when does $\mathcal{R}_{p, q}^{\sim}$ comprise a singleton only? Progress towards answers has been made in [13, 18]. Let $\phi_{p}$ denote product measure with density $p$ on $(\Omega, \mathcal{F})$. We define $\pi:[0,1] \times(0, \infty) \rightarrow[0,1]$ by

$$
\pi(p, q)=\frac{p}{p+q(1-p)} .
$$

When $p, q$ are given, we use the abbreviation $\pi=\pi(p, q)$. Note that $\pi \neq p$ except in the trivial cases $p=0, p=1$ or $q=1$.

Theorem 4.1 ([13]). (a) For $0 \leq p \leq 1$ and $q>0$, the set $\mathcal{R}_{p, q}^{0}$ of free random-cluster measures comprises the singleton $\phi_{\pi}$ only, where $\pi=\pi(p, q)$.

(b) For $0 \leq p \leq 1$ and $q>0$, the set $\mathcal{R}_{p, q}^{1}$ of wired random-cluster measures is nonempty. If $q \geq 1$, then $\mathcal{R}_{p, q}^{1}$ contains the weak limit $\phi_{p, q}^{1}$ given in (4.4) 
We present in Theorem 6.1 a) a necessary and sufficient condition for the statement $\phi_{\pi} \in \mathcal{R}_{p, q}^{\sim}$, for $\sim$ belonging to a certain class of equivalence relations to be defined in the next section.

We write $\{x \leftrightarrow y\}$ for the set of all $\omega \in \Omega$ for which there exists an open path joining vertex $x$ to vertex $y$. (For the moment, we refer to open paths in the undirected tree $T_{m}^{\prime}$.) The complement of the event $\{x \leftrightarrow y\}$ is denoted $\{x \leftrightarrow y\}$. We write $\{x \leftrightarrow \infty\}$ for the event that $x$ is the endvertex of an infinite open path of $T_{m}^{\prime}$. For a probability measure $\phi$ on $(\Omega, \mathcal{F})$, we define the percolation probability by

$$
\theta(\phi)=\phi(0 \leftrightarrow \infty)
$$

Of particular interest are the two special cases

$$
\theta^{0}(p, q)=\theta\left(\phi_{\pi}\right), \quad \theta^{1}(p, q)=\theta\left(\phi_{p, q}^{1}\right)
$$

where the former is well-defined for all $p, q$, and the latter when $q \geq 1$ at least.

The function $\theta^{0}(p, q)$ is the survival probability of a branching process with $\operatorname{bin}(m, \pi)$ family-sizes (subject to the small change of vertex degree at the origin). It may be computed as in (2.7) (2.8) In particular, its critical value

$$
p_{\mathrm{c}}^{0}(q)=\sup \left\{p: \theta^{0}(p, q)=0\right\}
$$

is immediately seen to be the value of $p$ for which $\pi(p, q)=m^{-1}$, whence

$$
p_{\mathrm{c}}^{0}(q)=\frac{q}{m+q-1} .
$$

Less standard is the calculation given in [13] of the critical value

$$
p_{\mathrm{c}}^{1}(q)=\sup \left\{p: \theta^{1}(p, q)=0\right\}
$$

when $q \geq 1$, namely

$$
p_{\mathrm{c}}^{1}(q)= \begin{cases}p_{\mathrm{c}}^{0}(q) & \text { if } 1 \leq q \leq 2 \\ U_{q} & \text { if } q>2\end{cases}
$$

where $U_{q}$ is the unique value of $p \in(0,1)$ for which the polynomial

$$
(q-1) x^{m+1}+\left(1-\frac{p}{1-p}-q\right) x^{m}+\frac{1}{1-p} x-1
$$

has a double root in $(0,1)$. Applying this as in [13] when $m=2$, we find that

$$
p_{\mathrm{c}}^{1}(q)= \begin{cases}\frac{q}{q+1} & \text { if } 1 \leq q \leq 2, \\ \frac{2 \sqrt{q-1}}{1+2 \sqrt{q-1}} & \text { if } q>2\end{cases}
$$

[An alternative proof of these facts may be obtained by the parallel/series replacement method used in the proof of Theorem 6.4, see [11].] 
We note from [13] for later use that, for $q \geq 1$,

$$
\theta^{1}(p, q)>0 \text { if and only if }\left\{\begin{array}{l}
1 \leq q \leq 2, p>p_{\mathrm{c}}^{1}(q), \text { or } \\
q>2, p \geq p_{\mathrm{c}}^{1}(q)
\end{array}\right.
$$

Let $q \geq 1$. It was proved in [13] that there exists a continuum of probability measures in $\mathcal{R}_{p, q}^{1}$ when $q>2$ and $p_{\mathrm{c}}^{1}(q) \leq p \leq p_{\mathrm{c}}^{0}(q)$, and it was conjectured that $\mathcal{R}_{p, q}^{1}$ contains exactly one measure when $p>p_{\mathrm{c}}^{0}(q)$. Uniqueness was proved in [18] for sufficiently large values of $p$, and we recall this result next. In the notation of Section 2 , we take as 'mother process' the process in which every individual has exactly $m$ children; the corresponding family-size probability generating function is given by $G(\alpha)=\alpha^{m}, \alpha \in \mathbb{R}$. On this graph, we construct bond percolation with density $\pi$ given in (4.7), and we ask for the probability that the origin is black. By Corollary 2.3 (see also [18]), the probability $\gamma$ that the origin is black satisfies

$$
\gamma=1 \text { if and only if } \pi \geq p_{\mathrm{b}}(m),
$$

where $p_{\mathrm{b}}(m)$ is given in $(2.12)$

Theorem 4.2 ([18]). Let $q \geq 1$ and let $p$ be such that

$$
\pi=\frac{p}{p+q(1-p)} \geq p_{\mathrm{b}}(m) .
$$

The set $\mathcal{R}_{p, q}^{1}$ comprises the singleton $\phi_{p, q}^{1}$ only.

Proof. This is a special case of the forthcoming Theorem 6.3 .

\section{Relations on the set of rays}

We consider next the case of a general boundary condition $\sim\left(\in \mathcal{E}_{\mathrm{m}}\right)$. We cannot prove in general that $\mathcal{R}_{p, q}^{\sim}$ is non-empty, but only for a certain class of equivalence relations which we introduce in this section. It is in fact unnecessary for the present purposes to require a relation on $\mathcal{R}$ to be an equivalence relation, and thus we shall broaden the discussion in this section to the class of all symmetric relations on $\mathcal{R}$.

For simplicity, we continue to consider the infinite $m$-ary tree $T_{m}^{\prime}$ with root 0 , and every edge oriented away from 0 . The conclusions of this section are valid under considerably less restrictive assumptions on the underlying tree.

We write $\mathcal{S}$ for the class of all symmetric reflexive relations on $\mathcal{R}$, and we think of a relation as a subset of the space $\mathcal{R}^{2}$. Thus we consider the set $\mathcal{S}$ of subsets $S$ of $\mathcal{R}^{2}$ such that:

(a) $(\rho, \rho) \in S$ for all $\rho \in \mathcal{R}$,

(b) $\left(\rho_{1}, \rho_{2}\right) \in S$ whenever $\left(\rho_{2}, \rho_{1}\right) \in S$.

The set $\mathcal{R}$ of rays may be viewed as a topological space with the product topology. Since $\mathcal{R}$ is the product of compact spaces, it is itself compact. The family $\left\{\mathcal{R}_{v}^{\prime}: v \in V\right\}$ 
forms a base for the space. We endow $\mathcal{R}^{2}$ with the product topology, and we call the relation $S \in \mathcal{S}$ closed (respectively, open, Borel) if $S$ is a closed (respectively, open, Borel) subset of $\mathcal{R}^{2}$. We write $\mathcal{S}_{\mathrm{c}}$ (respectively, $\mathcal{S}_{\mathrm{o}}, \mathcal{S}_{\mathrm{B}}$ ) for the set of closed (respectively, open, Borel) relations. By definition, $\mathcal{S}_{\mathrm{c}}, \mathcal{S}_{\mathrm{o}} \subseteq \mathcal{S}_{\mathrm{B}}$. We shall see in Corollary 5.6 that every open equivalence relation is closed.

There follows a description of a certain family of closed relations. A (finite or infinite) subset $W$ of $V$ is called incomparable if there exists no 0 -ray of $T_{m}^{\prime}$ which contains more than one member of $W ; W$ is called a cutset if it is incomparable and every 0-ray contains some member of $W$. The smallest cutset is the singleton set $\{0\}$. Let $W$ be a cutset. Each $\mathcal{R}_{w}^{\prime}, w \in W$, is an open subset of the compact space $\mathcal{R}$, and in addition $\left(\mathcal{R}_{w}^{\prime}: w \in W\right)$ is a cover of $\mathcal{R}$. By compactness, $W$ is finite. In summary, all cutsets are finite. Every cutset $W$ generates a finite set of vertices $\bar{W}=W \cup \operatorname{ins}(W)$, where ins $(W)$ is the set of all vertices $v \in V$ such that there exists a path of $T$ from 0 to $v$ which is vertex-disjoint from $W$. We refer to such a set $\bar{W}$ as a box of the tree.

Let $W$ be an incomparable (finite or infinite) set of vertices, and partition $W$ as $W=$ $W_{1} \cup W_{2}$. Let $S$ be the (equivalence) relation given by

$$
S=\left(\bigcup_{w \in W_{1}} \mathcal{R}_{w}^{\prime} \times \mathcal{R}_{w}^{\prime}\right) \cup\left(\bigcup_{w \in W_{2}}\left\{(\rho, \rho): \rho \in \mathcal{R}_{w}^{\prime}\right\}\right) \cup\left(\mathcal{R}_{W}^{\mathrm{c}} \times \mathcal{R}_{W}^{\mathrm{c}}\right),
$$

where

$$
\mathcal{R}_{W}^{\mathrm{c}}=\mathcal{R} \backslash \bigcup_{w \in W} \mathcal{R}_{w}^{\prime}
$$

In the usual jargon borrowed from the theory of electrical networks, the relation $S$ corresponds to 'wired boundary conditions' on $\mathcal{R}_{w}^{\prime}$ for every $w \in W_{1}$, 'free boundary conditions' on $\mathcal{R}_{w}^{\prime}$ for every $w \in W_{2}$, and a 'wired boundary condition' on the union of all other rays. Certainly $S$ is an equivalence relation, and in addition $S$ is a closed relation (it may be considered easier to see that $\mathcal{R}^{2} \backslash S$ is open). The construction of [13, Section 5] gives rise to equivalence relations of the above type. Note that the minimal and maximal relations $\sim^{0}$ and $\sim^{1}$ are both of this type (for $\sim^{1}$, take $W=\{0\}$ ); in particular, they are closed.

A simple example of the above recipe arises when $W$ is a cutset, and is therefore finite. Let $S^{W}$ be the relation:

$$
S^{W}=\bigcup_{w \in W} \mathcal{R}_{w}^{\prime} \times \mathcal{R}_{w}^{\prime}
$$

The equivalence classes of $S^{W}$ are the sets $\mathcal{R}_{w}^{\prime}, w \in W$. We call $S(\in \mathcal{S})$ a cutset relation if there exists a cutset $W$ such that $S=S^{W}$. The maximal equivalence relation $\sim^{1}$ is a cutset relation with single equivalence class $\mathcal{R}$.

We next continue the measurability discussions from Section 4. Recall first that, if $x \in V$ and $\pi$ is an infinite path of $T$ with endvertex $x$, then there exists a unique 0-ray, denoted $\rho_{\pi}$, such that $\pi$ and $\rho_{\pi}$ differ on only finitely many edges. (The path $\pi \in \Pi_{x}$ comprises a finite path from $x$ to some vertex $w$ directed in the opposite direction, followed by a ray in $\mathcal{R}_{w}$ which differs from $\rho_{\pi}$ only in the absence of the first section of $\rho_{\pi}$ 
from 0 to $w$.) For given $x$, the pair $\pi, \rho_{\pi}$ are in one-one correspondence. For $\pi$ a ray in $\mathcal{R}_{x}$, we denote $\rho_{\pi}$ by $\pi^{\prime}$. We consider five related definitions of subsets of the configuration space $\Omega$. Let $\Lambda$ be a finite subset of $V$.

1. $A_{u, v}^{S}$, for $u, v \in V$, is the set of configurations such that there exist open rays $\rho_{u} \in \mathcal{R}_{u}$, $\rho_{v} \in \mathcal{R}_{v}$ with $\left(\rho_{u}^{\prime}, \rho_{v}^{\prime}\right) \in S$. (These rays have endpoints $u, v$.)

2. $A_{u, v}^{S^{\prime}}$, for $u, v \in V$, is the set of configurations such that there exist open rays $\rho_{u} \in \mathcal{R}_{u}^{\prime}$, $\rho_{v} \in \mathcal{R}_{v}^{\prime}$ with $\left(\rho_{u}, \rho_{v}\right) \in S$. (These rays have common endpoint 0 .)

3. $K_{u, v, \Lambda}^{S}$, for $u, v \in \Lambda$, is the subset of $\Omega$ such that there exist infinite open vertexdisjoint paths $\pi(u) \in \Pi_{u}, \pi(v) \in \Pi_{v}$ satisfying $\left(\rho_{\pi(u)}, \rho_{\pi(v)}\right) \in S$ and that are edge-disjoint from $E_{\Lambda}$.

4. $K_{u, v}^{S}$, for $u, v \in V$, is similarly defined as the subset of $\Omega$ such that there exist vertexdisjoint open paths $\pi(u) \in \Pi_{u}, \pi(v) \in \Pi_{v}$ satsfying $\left(\rho_{\pi(u)}, \rho_{\pi(v)}\right) \in S$. Thus, $K_{u, v}^{S}=$ $K_{u, v,\{u, v\}}^{S}$.

5. $K_{e}^{S}$, for an edge $e=\langle u, v\rangle \in E$, equals $K_{u, v}^{S}=K_{u, v,\{u, v\}}^{S}$.

We say that two vertices $u, v$ are comparable if one is on the path from 0 to the other (including $u=0, v=0$ and $u=v$ ), and incomparable otherwise.

Theorem 5.1. The following are equivalent, for every $S \in \mathcal{S}$.

(i) $A_{u, v}^{S} \in \mathcal{F}$ for all $u, v \in V$.

(ii) $A_{u, v}^{S^{\prime}} \in \mathcal{F}$ for all $u, v \in V$.

(iii) $A_{u, v}^{S} \in \mathcal{F}$ for all incomparable $u, v \in V$.

(iv) $K_{u, v}^{S} \in \mathcal{F}$ for all $u, v \in V$.

(v) $K_{u, v, \Lambda}^{S} \in \mathcal{F}$ for all finite subsets $\Lambda \subseteq V$ and $u, v \in \Lambda$.

(vi) $K_{e}^{S} \in \mathcal{F}$ for all edges $e \in E$.

Proof. For two vertices $x, y \in V$, let $\pi_{x, y}$ be the path between $x$ and $y$, and let $P_{x, y}$ be the cylinder event that all edges in $\pi_{x, y}$ are open.

(i) $\Rightarrow$ (ii): $A_{u, v}^{S^{\prime}}=A_{u, v}^{S} \cap P_{0, u} \cap P_{0, v}$.

(ii) $\Rightarrow$ (iii): For $\omega \in \Omega$, let $\phi(\omega)$ equal $\omega$ except that all edges in $\pi_{0, u}$ and $\pi_{0, v}$ are open. Then $\phi: \Omega \rightarrow \Omega$ is measurable, and $A_{u, v}^{S}=\phi^{-1}\left(A_{u, v}^{S^{\prime}}\right)$.

(iii) $\Rightarrow$ (i): Suppose that $u$ lies between 0 and $v$. Let $W$ be the finite set of the possible vertices $w$ where a ray $\rho_{u}$ from $u$ that does not pass $v$ may first leave the path $\pi_{u, v}$. Then $A_{u, v}^{S}$ is the finite union of $P_{u, w} \cap A_{w, v}^{S}, w \in W$, and $P_{u, v} \cap B_{v}$, where $B_{v}$ is the event that there is an infinite open ray in $\mathcal{R}_{v}$.

(i) $\Rightarrow$ (iv): Considering the places where the paths $\pi_{u}$ and $\pi_{v}$ leave $\pi_{0, u}$ and $\pi_{0, v}$, we see that $K_{u, v}^{S}$ equals a finite union of sets $P_{u, x} \cap P_{v, y} \cap A_{x, y}^{S}$.

(iv) $\Rightarrow(v)$ : Define $\phi(\omega)$ to equal $\omega$ except that all edges in $E_{\Lambda}$ are deemed closed. Then $K_{u, v, \Lambda}^{S}=\phi^{-1}\left(K_{u, v}^{S}\right)$.

(v) $\Rightarrow$ (vi): A special case.

(vi) $\Rightarrow$ (iii): Let $w$ be the father of $v$. Define $\phi(\omega)$ to equal $\omega$ except that all edges in $\pi_{u, w}$ are deemed open, and all edges that are incident to this path, except at $u$, are closed. Then $A_{u, v}^{S}=\phi^{-1}\left(K_{\langle w, v\rangle}^{S}\right)$. 
We say that the relation $S$ is measurable if the equivalent conditions in Theorem 5.1 are satisfied. Note that this agrees with the previous use of the word 'measurable' as applied to equivalence relations. We now investigate measurability further. Let $O \subseteq$ $\mathcal{R} \times \Omega$ be the set of pairs $(\rho, \omega)$ such that $\rho$ is a 0 -ray that is open in $\omega$; this is a closed subset of the compact space $\mathcal{R} \times \Omega$. Further, let $D \subseteq \mathcal{R} \times \mathcal{R} \times \Omega$ be the set of triples $\left(\rho_{1}, \rho_{2}, \omega\right)$ such that both $\rho_{1}$ and $\rho_{2}$ are open in $\omega$, and $\left(\rho_{1}, \rho_{2}\right) \in S$. Thus,

$$
D=(\mathcal{R} \times O) \cap(\widetilde{\mathcal{R} \times O}) \cap(S \times \Omega),
$$

where $\tilde{A}=\left\{\left(\rho_{1}, \rho_{2}, \omega\right):\left(\rho_{2}, \rho_{1}, \omega\right) \in A\right\}$. Hence, $D$ is a closed, and thus compact, subset of $\mathcal{R} \times \mathcal{R} \times \Omega$ if $S$ is closed, and $D$ is Borel if $S$ is Borel. We can now state the following links between the measurability of a relation $S$ and the properties of $S$ viewed as a subset of $\mathcal{R}^{2}$.

Theorem 5.2. (a) A closed relation is measurable.

(b) A measurable relation is Borel.

Proof. (a) If $u, v \in V$, then $A_{u, v}^{S^{\prime}}=\pi_{3}\left(D \cap\left(\mathcal{R}_{u}^{\prime} \times \mathcal{R}_{v}^{\prime} \times \Omega\right)\right)$, where $\pi_{3}$ denotes the projection on the third factor. If $S$ is closed, this is the projection of a compact set, and thus compact, so (ii) in Theorem 5.1 is satisfied. (This part is also an immediate consequence of Theorem 5.4 below.)

(b) Let $\psi: \mathcal{R}^{2} \rightarrow \Omega$ be defined by $\psi\left(\rho_{1}, \rho_{2}\right)(e)=1$ if and only if $e \in \rho_{1} \cup \rho_{2}$. In other words, $\psi\left(\rho_{1}, \rho_{2}\right)$ is the configuration with all edges in $\rho_{1}$ and $\rho_{2}$ open, but no others. The function $\psi$ is continuous, and thus (Borel) measurable.

Let $S$ be a measurable relation, so that each of the six parts of Theorem 5.1 is valid. Two distinct 0-rays $\rho_{1}$ and $\rho_{2}$ pass though two incomparable vertices $u$ and $v$, and they satisfy $\left(\rho_{1}, \rho_{2}\right) \in S$ if and only if $\rho_{1} \in \mathcal{R}_{u}^{\prime}, \rho_{2} \in \mathcal{R}_{v}^{\prime}$, and $\psi\left(\rho_{1}, \rho_{2}\right) \in A_{u, v}^{S^{\prime}}$. Hence,

$$
S=\left\{\bigcup_{u, v \text { incomparable }}\left(\mathcal{R}_{u}^{\prime} \times \mathcal{R}_{v}^{\prime}\right) \cap \psi^{-1}\left(A_{u, v}^{S^{\prime}}\right)\right\} \cup\{(\rho, \rho): \rho \in \mathcal{R}\},
$$

which is a Borel subset of $\mathcal{R}^{2}$, using (ii) in Theorem 5.1

Theorem 5.2 leaves an obvious gap.

Problem 5.3. Is every Borel relation measurable?

Note that the proof of Theorem 5.2 a) breaks down for Borel relations because the projection of a Borel set is in general not a Borel set. However, for Polish spaces (and the spaces we consider are such), the projection of a Borel set is a Suslin (or analytic) set, and such sets are universally measurable, that is, they belong to the completion $\mathcal{F}_{\mu}$ of $\mathcal{F}$ for every finite Borel measure $\mu$. (See, for example, [6, Sections 8.2, 8.4].) Theorem 5.1 holds also if we replace $\mathcal{F}$ by the $\sigma$-field $\bigcap_{\mu} \mathcal{F}_{\mu}$ of universally measurable sets, and we thus see that every Borel relation is (at least) universally measurable, which is enough for the definition (4.6) to make sense.

The closed relations have a certain property that will enable a large-volume limit for random-cluster measures, and we present this next. For any box $\Lambda$ and any $\omega \in \Omega$, we 
write $\omega_{\Lambda}^{1}$ for the configuration that agrees with $\omega$ on $E_{\Lambda}$ and equals 1 elsewhere, which is to say that

$$
\omega_{\Lambda}^{1}(e)= \begin{cases}\omega(e) & \text { if } e \in E_{\Lambda} \\ 1 & \text { otherwise }\end{cases}
$$

We define the event

$$
\bar{K}_{e, \Lambda}^{S}=\left\{\omega \in \Omega: \omega_{\Lambda}^{1} \in K_{e}^{S}\right\}
$$

i.e., if $e=\langle u, v\rangle$, the set of configurations such that there exist vertex-disjoint paths $\pi(u) \in \Pi_{u}, \pi(v) \in \Pi_{v}$ with $\left(\rho_{\pi(u)}, \rho_{\pi(v)}\right) \in S$ such that the parts of the paths inside $\Lambda$ are open. Note that $\bar{K}_{e, \Lambda}^{S}$ is a cylinder event, and that it is decreasing in $\Lambda$.

Theorem 5.4. Let $S \in \mathcal{S}$. Then

$$
\text { for all } e \in E, \quad \bar{K}_{e, \Lambda}^{S} \downarrow K_{e}^{S} \quad \text { as } \Lambda \uparrow V,
$$

if and only if $S$ is closed.

Proof. Assume first that $S$ is closed. Let $e=\langle x, y\rangle \in E$ and $\omega \in \Omega$.

Let $\Pi_{x}^{e}$ (respectively, $\Pi_{y}^{e}$ ) be the set of infinite (undirected) paths of $E \backslash\{e\}$ with endpoint $x$ (respectively, $y$ ), and let $F_{\Lambda}=F_{\Lambda}(\omega)$ be the set of all pairs $\left(\rho_{1}, \rho_{2}\right) \in S$ such that:

(i) $\rho_{1}=\rho_{\nu(x)}$ for some $v(x) \in \Pi_{x}^{e}$,

(ii) $\rho_{2}=\rho_{\nu(y)}$ for some $v(y) \in \Pi_{y}^{e}$,

(iii) all edges in $v(x), v(y)$ which belong to $E_{\Lambda}$ are open.

Then $F_{\Lambda}$ is the intersection of $S$ with a product of two closed sets of rays, and is therefore closed and hence compact. Furthermore, $F_{\Lambda}$ is decreasing in $\Lambda$.

We similarly define $F=F(\omega)$ by (i) and (ii), but replacing (iii) by:

(iii') all edges in $v(x), v(y)$ are open.

Since $v(x)$ and $v(y)$ are uniquely determined by $\rho_{1}$ and $\rho_{2}$, it is clear that

$$
F=\bigcap_{\Lambda} F_{\Lambda}
$$

Since the sets are compact, this implies that

$$
F(\omega) \neq \emptyset \Leftrightarrow F_{\Lambda}(\omega) \neq \emptyset \text { for every } \Lambda .
$$

We now observe that $\omega \in \bar{K}_{e, \Lambda}^{S} \Leftrightarrow F_{\Lambda}(\omega) \neq \emptyset$, and $\omega \in K_{e}^{S} \Leftrightarrow F(\omega) \neq \emptyset$. Thus (5.2) can be written $K_{e}^{S}=\bigcap_{\Lambda} \bar{K}_{e, \Lambda}^{S}$, which is (5.1)

Suppose conversely that $(5.1)$ holds. Let $\left(\rho_{1}^{n}, \rho_{2}^{n}\right), n \geq 1$, be a sequence in $S$ such that

$$
\left(\rho_{1}^{n}, \rho_{2}^{n}\right) \rightarrow\left(\rho_{1}, \rho_{2}\right) \quad \text { as } n \rightarrow \infty
$$


for some $\rho_{1}, \rho_{2} \in \mathcal{R}$ with $\rho_{1} \neq \rho_{2}$. We shall show that $\left(\rho_{1}, \rho_{2}\right) \in S$. Let $0, x_{1}, x_{2}, \ldots, x_{m}$ be the vertices, taken in order, in the (finite) intersection of $\rho_{1}$ and $\rho_{2}$, and write $x=x_{m}$, write $y$ for the next vertex on $\rho_{2}$ as one moves from $x_{m}$ towards infinity, and let $e=\langle x, y\rangle$.

Let $\omega \in \Omega$ be the configuration which takes the value 1 on edges $f \in \rho_{1} \cup \rho_{2}$, and the value 0 on all other edges.

Write the edges of $\rho_{j}^{n}$ in order as $f_{j}^{n}(1), f_{j}^{n}(2), \ldots$, and those of $\rho_{j}$ as $f_{j}(1), f_{j}(2), \ldots$ By (5.3) for $j=1,2$, for $i \geq 1$, and for all large $n$, we have $f_{j}^{n}(i)=f_{j}(i)$. Therefore, for all boxes $\Lambda$, and for $j=1,2$ and all large $n$, the intersection of $E_{\Lambda}$ with $\rho_{j}^{n}$ equals its intersection with $\rho_{j}$. By the assumption that $\left(\rho_{1}^{n}, \rho_{2}^{n}\right) \in S$ for each $n$, we find that $\omega \in \bar{K}_{e, \Lambda}^{S}$ for all $\Lambda$, and therefore, by (5.1).

$$
\omega \in \lim _{\Lambda \uparrow V} \bar{K}_{e, \Lambda}^{S}=K_{e}^{S}
$$

Since the only open rays in $\omega$ are the subrays of $\rho_{1}$ and $\rho_{2}$, this can happen only if $\left(\rho_{1}, \rho_{2}\right) \in S$. Therefore $S$ is closed, as required.

We return now to the universe of equivalence relations on $\mathcal{R}$, which we think of as binary relations and denote by $\sim$. We call $\sim \in \mathcal{E}$ closed (respectively, open) if it is closed (respectively, open) when viewed as a relation. The set of closed (respectively, open) equivalence relations is denoted $\mathcal{E}_{\mathrm{c}}$ (respectively, $\mathcal{E}_{\mathrm{o}}$ ). By definition, $\mathcal{E}_{\mathrm{c}} \subseteq \mathcal{S}_{\mathrm{c}}$ and $\mathcal{E}_{\mathrm{o}} \subseteq \mathcal{S}_{\mathrm{o}}$. Let $\mathcal{E}_{\text {cut }}\left(\subseteq \mathcal{E}_{\mathrm{o}}\right)$ be the set of equivalence relations which, when viewed as relations, are cutset relations. The word 'measurable' applied to an equivalence relation has been explained in Section 4 and elaborated in Theorem 5.1. We continue with a theorem concerning open equivalence relations.

Theorem 5.5. Let $\sim$ be an equivalence relation on $\mathcal{R}$, and let $\mathcal{R}(k)$ be the set of all paths of length $k$ starting at 0 . The following are equivalent.

(a) $\sim$ is open.

(b) Each equivalence class of $\sim$ is an open subset of $\mathcal{R}$.

(c) There exists an integer $k \geq 1$, and an equivalence relation $\sim_{k}$ on $\mathcal{R}(k)$, such that $\sim$ is specified by $\sim_{k}$ in the sense that:

$$
\rho_{1} \sim \rho_{2} \quad \text { if and only if } \rho_{1}(k) \sim_{k} \rho_{2}(k),
$$

where $\rho(k)$ denotes the path comprising the first $k$ edges of an infinite path $\rho$ from 0.

Proof. Suppose that $\sim \in \mathcal{E}_{\mathrm{o}}$. Then the sections $A_{\rho}=\left\{\rho^{\prime} \in \mathcal{R}: \rho \sim \rho^{\prime}\right\}, \rho \in \mathcal{R}$, are open, and thus the equivalence classes are open.

Suppose next that (b) holds. The equivalence classes cover the compact space $\mathcal{R}$, whence there exists a finite subcover. Since the equivalence classes are disjoint, no proper subset covers $\mathcal{R}$, and therefore there exist only finitely many equivalence classes. Each class is the complement of the union of the others, and is therefore closed and thus compact. Being open, each class is a union of sets of the form $\mathcal{R}_{v}^{\prime}, v \in V$ (since these form a base), and, being compact, is a finite union of such sets. There exists therefore an integer 
$k$ such that every equivalence class is a union of sets of the form $\mathcal{R}_{w}^{\prime}$ as $w$ ranges over the set of vertices at distance $k$ from the root 0 .

That (c) implies (a) is obvious.

Corollary 5.6. Every open equivalence relation is closed, and thus measurable.

Proof. Let $\sim \in \mathcal{E}_{\mathrm{O}}$. By Theorem 5.5, the set $\left\{\left(\rho, \rho^{\prime}\right) \in \mathcal{R}^{2}: \rho \sim \rho^{\prime}\right\}$ is a finite union of closed sets and is therefore closed.

We finish this section with a note. There are of course many equivalence relations which are not closed. However, with each relation $\sim$ may be associated a closed relation, termed the closure of $\sim$ and denoted $\sim$. We define $\sim$ to be the intersection of all closed equivalence relations $\sim^{\prime}$ satisfying $\sim \leq \sim^{\prime}$ with respect to the partial order of (4.1). It is easily checked that $\sim$ is itself a closed equivalence relation.

\section{Random-cluster measures with general boundary conditions}

Let us consider the tree $T=T_{m}^{\prime}$ with $m \geq 2$. We show first that $\mathcal{R}_{p, q}^{\sim} \neq \emptyset$ for $\sim \in \mathcal{E}_{\mathrm{c}}$ and $q \geq 1$. The proofs are given later in the section, and do not appear to extend to the case $q<1$.

Theorem 6.1. Let $\sim \in \mathcal{E}_{\mathrm{c}}$ and let $0 \leq p \leq 1$.

(a) Let $\pi$ be given by (4.7), and suppose $p \neq 1, q \neq 1$. The product measure $\phi_{\pi}$ satisfies $\phi_{\pi} \in \mathcal{R}_{p, q}^{\sim}$ if and only if

$\phi_{\pi}$ (there exist two or more equivalent open rays $)=0$.

(b) If $q \geq 1$, the weak limit

$$
\phi_{p, q}^{1, \sim}=\lim _{\Lambda \uparrow V} \phi_{\Lambda, p, q}^{1, \sim}
$$

exists and satisfies $\phi_{p, q}^{1, \sim} \in \mathcal{R}_{p, q}^{\sim}$.

(c) Let $q \geq 1$ and let $\phi \in \mathcal{R}_{p, q}^{\sim}$. Then

$$
\phi_{\pi} \leq_{\mathrm{st}} \phi \leq_{\mathrm{st}} \phi_{p, q}^{1, \sim} \leq_{\mathrm{st}} \phi_{p, q}^{1} .
$$

(d) Let $q \geq 1$ and let $p$ be such that $\theta^{1}(p, q)=0$; see (4.12) The set $\mathcal{R}_{p, q}^{\sim}$ comprises the singleton measure $\phi_{\pi}$ only.

More generally, the proof of (c) shows that $\phi_{p, q}^{1, \sim} \leq_{\text {st }} \phi_{p, q}^{1, \sim^{\prime}}$ whenever $\sim \leq \sim^{\prime}$. Part (b) may be extended as follows to arbitrary equivalence relations.

Theorem 6.2. Let $\sim \in \mathcal{E}$, and let $\sim$ be the closure of $\sim$. If $q \geq 1$, then

$$
\phi_{\Lambda, p, q}^{1, \sim} \Rightarrow \phi_{p, q}^{1, \widetilde{\sim}} \quad \text { as } \Lambda \uparrow V .
$$


This leads to the question: for $\sim \in \mathcal{E}_{\mathrm{m}}$, is $\phi_{p, q}^{1, \bar{q}}$ a $(\sim)$-random-cluster measure? The answer can be positive or negative, as illustrated by the following examples.

1. Let $\sim^{\prime}$ be a closed relation with some equivalence class $C$ satisfying $|C| \geq 2$, and let $\rho \in C$. Let $\sim$ be the relation having the same equivalence classes as $\sim^{\prime}$ except that $C$ is replaced by the two equivalence classes $C \backslash\{\rho\}$ and $\{\rho\}$. It is easily seen that $\sim$ and $\sim^{\prime}$ $(=\approx)$ generate the same family of random-cluster measures, and hence $\phi_{p, q}^{1, \sim} \in \mathcal{R}_{p, q}^{\sim}$ by (6.1). (We return to such constructions after Theorem 6.4.)

2. Consider for definiteness the tree $T_{2}^{\prime}$. Each of the three subtrees with root 0 is a binary tree, and each vertex therein may be viewed as a combination of leftward and rightward steps from 0 . Let $\mathcal{T}$ be the set of all (infinite) 0-rays which have only finitely many leftward steps. The set $\mathcal{T}$ is dense in $\mathcal{R}$, and is countable and hence Borel. Let $\sim$ be the equivalence relation whose unique non-trivial equivalence class is $\mathcal{T}$. It is easily seen that $\widetilde{\sim}=\sim^{1}$. Let $q \geq 1, p<1$, and $\phi \in \mathcal{R}_{p, q}^{\sim}$. By stochastic domination, $\phi \leq_{\text {st }} \phi_{p}$, and therefore, since $\mathcal{T}$ is countable,

$$
\phi(\text { some ray in } \mathcal{T} \text { is open })=0 .
$$

This implies that, with $\phi$-probability 1 , there is no pair of distinct equivalent open rays in the tree $T_{2}^{\prime}$. By the forthcoming Lemma 6.5(a), the unique $(\sim)$-random-cluster measure is the product measure $\phi_{\pi}$. By (6.1)

$$
\phi_{p, q}^{1, \bar{\sim}}=\phi_{p, q}^{1}=\lim _{\Lambda \uparrow V} \phi_{\Lambda, p, q}^{1, \sim} \neq \phi_{\pi}
$$

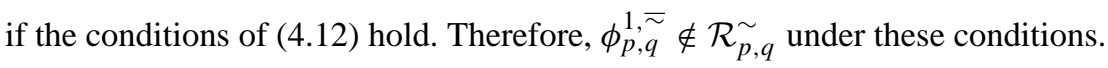

We turn next to the question of the uniqueness of random-cluster measures for large $p$. We shall prove that $\mathcal{R}_{p, q}^{\sim}$ is a singleton when $\sim \in \mathcal{E}_{\mathrm{o}}, q \geq 1$, and $p$ is sufficiently large. This extends [18, Thm 1.3], which was concerned with the wired (maximal) boundary condition. We leave as a problem the question whether this result extends to arbitrary closed relations.

Theorem 6.3. Let $\sim \in \mathcal{E}_{\mathrm{o}}$ and let $0 \leq p \leq 1, q \geq 1$. If $\pi \geq p_{\mathrm{b}}(m)$ where $\pi$ and $p_{\mathrm{b}}(m)$ are given in (4.7) and (2.12) then $\mathcal{R}_{p, q}^{\sim}$ comprises the singleton $\phi_{p, q}^{1, \sim}$ only.

Finally, we turn to the question of the degree to which the measure $\phi_{p, q}^{1, \sim}$ characterises the relation $\sim$, and for simplicity we consider first the case when $\sim$ is a cutset relation. Suppose that $\sim \in \mathcal{E}_{\text {cut }}$, which is to say that $\sim=\sim{ }^{W}$ for some cutset $W$. Let $T_{W}$ be the set of edges $e=\langle u, v\rangle$ which belong to no ray in $\bigcup_{w \in W} \mathcal{R}_{w}$. It is an easy consequence of the forthcoming Lemma 6.5 that any member $\phi$ of $\mathcal{R}_{p, q}^{\sim}$ may be written in the form

$$
\phi=\left\{\prod_{e \in T_{W}} \phi_{e, \pi}\right\} \times\left\{\prod_{w \in W} \phi_{w, p, q}\right\},
$$

where $\phi_{e, \pi}$ is the Bernoulli measure on $\{0,1\}$ associated with the state of $e$, and $\phi_{w, p, q}$ is a wired random-cluster measure on the graph induced by $\mathcal{R}_{w}$ with root $w$; conversely, 
any such $\phi$, with any choice of wired random-cluster measures $\phi_{w, p, q}$, belongs to $\mathcal{R}_{p, q}^{\sim}$. In other words, $\phi \in \mathcal{R}_{p, q}^{\sim}$ may be described as product measure on $T_{W}$ with density $\pi$, combined with a wired random-cluster measure on the graph formed by the rays in each given $\mathcal{R}_{w}, w \in W$. In particular,

$$
\phi_{p, q}^{1, \sim}=\left\{\prod_{e \in T_{W}} \phi_{e, \pi}\right\} \times\left\{\prod_{w \in W} \phi_{w, p, q}^{1}\right\},
$$

where $\phi_{w, p, q}^{1}$ is the maximal wired random-cluster measure on $\mathcal{R}_{w}$.

We say that $\phi_{w, p, q}^{1}$ possesses a product component if there exists a non-empty subset $F$ of the set $E\left(\mathcal{R}_{w}\right)$ of edges of $\mathcal{R}_{w}$ such that

$$
\phi_{w, p, q}^{1}=\left\{\prod_{f \in F} \phi_{f, \pi}\right\} \times \psi
$$

for some probability measure $\psi$ on the set of configurations of $E\left(\mathcal{R}_{w}\right) \backslash F$. When $q>1$ and $\theta^{1}(p, q)>0$ then, by the results of [13], or as a consequence of Lemma 6.5. b) below, $\phi_{w, p, q}^{1}$ possesses no product component. It follows by (6.3) that, for any cutset relation $\sim^{W}$, the measure $\phi_{p, q}^{1, \sim}$ is characterised by the set $W$. That is,

$$
\phi_{p, q}^{1, \sim} \neq \phi_{p, q}^{1, \sim^{\prime}} \quad \text { for } \sim, \sim^{\prime} \in \mathcal{E}_{\text {cut }}, \sim \neq \sim^{\prime},
$$

whenever $q>1$ and $(4.12)$ holds.

Note in passing that $\mathcal{R}_{p, q}^{\sim}$ contains a continuum of distinct measures whenever there are a continuum of distinct wired measures on the graph induced by any given $\mathcal{R}_{w}$. See [13, Section 5].

The above conclusion is extended to open equivalence relations in the next theorem.

Theorem 6.4. Let $q>1$ and let $p$ be such that $p \neq 1$ and $\theta^{1}(p, q)>0$; see (4.12) For distinct members $\sim, \sim^{\prime}$ of $\mathcal{E}_{\mathrm{o}}$, we have $\phi_{p, q}^{1, \sim} \neq \phi_{p, q}^{1, \sim^{\prime}}$.

The conclusion of this theorem is false with $\mathcal{E}_{\mathrm{o}}$ replaced by $\mathcal{E}_{\mathrm{c}}$. For example, let $W$ be a cutset and let $w \in W$. Consider the closed (equivalence) relation $S$ given by

$$
S=\left\{\bigcup_{x \in W, x \neq w} \mathcal{R}_{x}^{\prime} \times \mathcal{R}_{x}^{\prime}\right\} \cup\left\{(\rho, \rho): \rho \in \mathcal{R}_{w}^{\prime}\right\}
$$

Now let $\rho, \rho^{\prime} \in \mathcal{R}_{w}^{\prime}$ be distinct, and let $S^{\prime}=S \cup\left\{\left(\rho, \rho^{\prime}\right),\left(\rho^{\prime}, \rho\right)\right\}$. Then $S$ and $S^{\prime}$ are closed equivalence relations which generate the same family of random-cluster measures.

The proofs of the three theorems above are preceded by a lemma, a special case of which may be found in [13]; see also [7]. We let $J_{e}=\{e$ is open $\}$. The $\sigma$-field generated by the states of edges $f \in E$ with $f \neq e$ is denoted $\mathcal{T}_{e}$. The event $K_{e}^{\sim}$ is defined prior to Theorem 5.1. 
Lemma 6.5. Let $\sim \in \mathcal{E}_{\mathrm{m}}$ and $0 \leq p \leq 1, q>0$.

(a) A probability measure $\phi$ on $(\Omega, \mathcal{F})$ satisfies $\phi \in \mathcal{R}_{p, q}^{\sim}$ if and only if, for all e $\in E$ and for $\phi$-almost every $\xi$,

$$
\phi\left(J_{e} \mid \mathcal{T}_{e}\right)(\xi)= \begin{cases}p & \text { if } \xi \in K_{e}^{\sim}, \\ \pi & \text { if } \xi \notin K_{e}^{\sim},\end{cases}
$$

where $\pi$ is given in (4.7)

(b) Let $e \in E$ and $\phi \in \mathcal{R}_{p, q}^{\sim}$ where $p \neq 0,1$ and $q \neq 1$. The (random) state of $e$ is independent of the states of $E \backslash\{e\}$, equalling 1 with probability $\pi$, if and only if

$$
\phi\left(K_{e}^{\sim}\right)=0 .
$$

Proof. (a) By an application of [19, Propn IV.1.8], the random-cluster measure $\phi_{G, p, q}$ on a finite graph $G=(V, E)$ is characterised by the statement that, for all edges $e=$ $\langle x, y\rangle \in E$,

$$
\phi_{G, p, q}\left(J_{e} \mid \mathcal{T}_{e}\right)(\xi)= \begin{cases}p & \text { if } \xi \in K_{e}, \\ \pi & \text { if } \xi \notin K_{e},\end{cases}
$$

where $K_{e}$ is the event that $x$ and $y$ are joined by an open path of $E \backslash\{e\}$. See [11, Thm $2.1]$, for example. If $\phi$ satisfies the condition of the lemma then, by (4.2), $\phi \in \mathcal{R}_{p, q}^{\sim}$. The converse is similar.

(b) When $p \neq 0,1$ and $q \neq 1$, we have $p \neq \pi$, and the claim follows by part (a).

Proof of Theorem 6.1. (a) Suppose $p \neq 0$; the case $p=0$ is trivial. If $\phi_{\pi}$ satisfies the condition, then $\phi_{\pi}\left(K_{e}^{\sim}\right)=0$ for all $e \in E$, implying that $\phi_{\pi}$ satisfies (6.5). By Lemma $6.5(\mathrm{a}), \phi_{\pi} \in \mathcal{R}_{p, q}^{\sim}$. The converse argument is valid when $p \neq \pi$, which requires that $p \neq 1, q \neq 1$.

(b) Let $\sim \in \mathcal{E}_{\mathrm{c}}$. By the FKG inequality in the usual way (see [8, Thm 3.1(a)] for example), the limit

$$
\phi=\lim _{\Lambda \uparrow V} \phi_{\Lambda, p, q}^{1, \sim}
$$

exists and is a probability measure. We prove next that $\phi$ satisfies (6.5) for all $e$ and $\phi$-almost every $\xi$, and the claim will follow.

For $\xi \in \Omega$ and $F \subseteq E$, write $[\xi]_{F}$ for the set of all configurations which agree with $\xi$ on $F$. For $W \subseteq V$ and $e \in E_{W}$, let $[\xi]_{W}$ (respectively, $[\xi]_{W \backslash e}$ ) be an abbreviation for $[\xi]_{E_{W}}$ (respectively, $[\xi]_{E_{W} \backslash\{e\}}$ ). We write $\xi_{W}^{1}$ for the configuration which agrees with $\xi$ on $E_{W}$ and which equals 1 elsewhere. For economy of notation, we shall omit explicit reference to the values of $p$ and $q$ in the rest of this proof, and thus we write $\phi_{\Lambda}^{1}=\phi_{\Lambda, p, q}^{1, \sim}$. 
By the martingale convergence theorem, for $e=\langle x, y\rangle \in E$ and $\phi$-almost every $\xi$,

$$
\begin{aligned}
\phi\left(J_{e} \mid \mathcal{T}_{e}\right)(\xi) & =\lim _{\Lambda \uparrow V} \frac{\phi\left(J_{e},[\xi]_{\Lambda \backslash e}\right)}{\phi\left([\xi]_{\Lambda \backslash e}\right)} \\
& =\lim _{\Lambda \uparrow V} \lim _{\Delta \uparrow V} \frac{\phi_{\Delta}^{1}\left(J_{e},[\xi]_{\Lambda \backslash e}\right)}{\phi_{\Delta}^{1}\left([\xi]_{\Lambda \backslash e}\right)} \\
& =\lim _{\Lambda \uparrow V} \lim _{\Delta \uparrow V} \phi_{\Delta}^{1}\left(\phi_{\Delta}^{1}\left(J_{e} \mid[\xi]_{\Delta \backslash e}\right) \mid[\xi]_{\Lambda \backslash e}\right) \\
& =\lim _{\Lambda \uparrow V} \lim _{\Delta \uparrow V} \phi_{\Delta}^{1}\left(g_{\Delta} \mid[\xi]_{\Lambda \backslash e}\right),
\end{aligned}
$$

by (6.6), where

$$
g_{\Delta}(\xi)=\pi+(p-\pi) 1_{\bar{K}_{e, \Delta}^{\sim}}^{\sim}(\xi)
$$

and $\bar{K}_{e, \Delta}^{\sim}=\left\{\omega \in \Omega: \omega_{\Delta}^{1} \in K_{e}^{\sim}\right\}$. Here and later, $1_{A}$ denotes the indicator function of the event $A$. Since $\sim$ is closed by assumption, we find by Theorem 5.4 that

$$
\bar{K}_{e, \Delta}^{\sim} \downarrow K_{e}^{\sim} \quad \text { as } \Delta \uparrow V,
$$

whence $g_{\Delta} \downarrow g$ where $g=\pi+(p-\pi) 1_{K_{e}^{\sim}}$.

We claim that

$$
\phi_{\Delta}^{1}\left(g_{\Delta} \mid[\xi]_{\Lambda \backslash e}\right) \rightarrow \phi\left(g \mid[\xi]_{\Lambda \backslash e}\right) \quad \text { as } \Delta \uparrow V,
$$

and we prove this as follows. Let $\Delta^{\prime}$ be a box satisfying $\Lambda \subseteq \Delta^{\prime} \subseteq \Delta$, and write $\psi_{\Delta}(\cdot)=$ $\phi_{\Delta}^{1}\left(\cdot \mid[\xi]_{\Lambda \backslash e}\right)$. Since $g_{\Delta}$ is non-increasing in $\Delta$,

$$
\psi_{\Delta}(g) \leq \psi_{\Delta}\left(g_{\Delta}\right) \leq \psi_{\Delta}\left(g_{\Delta^{\prime}}\right) .
$$

We take the limits as $\Delta \uparrow V$ and $\Delta^{\prime} \uparrow V$, in that order, and we appeal to the dominated convergence theorem to deduce (6.9)

By the martingale convergence theorem,

$$
\phi\left(g \mid[\xi]_{\Lambda \backslash e}\right) \rightarrow g(\xi) \quad \text { as } \Lambda \uparrow V \text {, for } \phi \text {-almost every } \xi \text {. }
$$

Hence, (6.7) and (6.9) show that (6.5) holds, and the result follows.

(c) Let $A$ be an increasing cylinder event in $\mathcal{F}$, and suppose that $A$ is defined on a finite set $B$ of edges. Let $\Lambda$ be a box of the tree satisfying $E_{\Lambda} \supseteq B$. In the construction of $\phi_{\Lambda, p, q}^{\xi, \sim}$ in Section 4, we add a certain set of (permanently open) new edges to $\Lambda$; for $\phi_{\Lambda, p, q}^{1, \sim}$ we add a larger (or equal) set of new edges and for $\phi_{\Lambda, p, q}^{1}$ an even larger set; on the other hand, no such edges are added for $\phi_{\Lambda, p, q}^{0}=\phi_{\Lambda, p, q}^{0, \sim_{0}}$. By the FKG inequality, the addition of a new open edge gives rise to a stochastically larger random-cluster measure. Thus, for every $\xi$,

$$
\phi_{\Lambda, p, q}^{0}(A) \leq \phi_{\Lambda, p, q}^{\xi, \sim}(A) \leq \phi_{\Lambda, p, q}^{1, \sim}(A) \leq \phi_{\Lambda, p, q}^{1}(A) .
$$


Here, $\phi_{\Lambda, p, q}^{0}$ is the product measure $\phi_{\pi}$ (cf. Theorem 4.1 a)). Using (4.6) and taking the expectation over $\xi$ we find

$$
\phi_{\pi}(A) \leq \phi(A) \leq \phi_{\Lambda, p, q}^{1, \sim}(A) \leq \phi_{\Lambda, p, q}^{1}(A)
$$

Now let $\Lambda \uparrow V$.

(d) Let $\phi \in \mathcal{R}_{p, q}^{\sim}$. Since $\theta^{1}(p, q)=0$, there is $\phi_{p, q}^{1}$-a.s. no open ray. By (c), there is thus $\phi$-a.s. no open ray, and thus, by (4.2) for every cylinder set $A$ defined on some finite edge-set $B$ and every box $\Lambda$ with $E_{\Lambda} \supseteq B$,

$$
\phi_{\Lambda, p, q}^{\xi, \sim}(A)=\phi_{\Lambda, p, q}^{\xi, \sim 0}(A)=\phi_{\pi}(A) \quad \text { for } \phi \text {-a.e. } \xi .
$$

Hence, by (4.6) and taking the expectation, $\phi(A)=\phi_{\pi}(A)$.

Proof of Theorem 6.2. Let $\sim \in \mathcal{E}$. Using (4.5) in the usual way, we may restrict ourselves for simplicity to sets $\Lambda$ which are boxes. Let $\Lambda$ be a finite box of $V$. For $v \in \Lambda$, let $C_{v}$ be the subset of $\mathcal{R}$ containing all 0 -rays whose final point of intersection with $\Lambda$ is $v$. Let $\approx$ be the lowest equivalence relation (in the sense of the natural partial order on equivalence relations, see (4.1) on the $C_{v}$ such that: $C_{v} \approx C_{w}$ if there exist $\rho_{v} \in C_{v}, \rho_{w} \in C_{w}$ such that $\rho_{v} \sim \rho_{w}$. The relation $\approx$ may be extended without change of notation to an equivalence relation on $\mathcal{R}$. By construction, $\approx$ is closed (since each $C_{v}$ is closed). Since $\sim \leq \approx$, we have

$$
\sim \leq \bar{\sim} \approx
$$

Let $\omega \in \Omega$, and let $k^{1, \sim}(\Lambda, \omega)$ be given as before (4.2). We claim that

$$
k^{1, \sim}(\Lambda, \omega)=k^{1, \approx}(\Lambda, \omega) .
$$

By (6.10) $(6.11) k^{1, \sim}(\Lambda, \omega)=k^{1, \widetilde{\sim}}(\Lambda, \omega)$, and hence $\phi_{\Lambda, p, q}^{1, \sim}=\phi_{\Lambda, p, q}^{1, \bar{\sim}}$. The claim of the theorem follows on taking the limit $\Lambda \uparrow V$.

We prove (6.11) next. Let $A^{\sim}=A^{\sim}(\Lambda, \omega)$ be the set of edges which are added to $G(\Lambda, \omega)$ in the construction prior to (4.2) of the measure $\phi_{\Lambda, p, q}^{1, \sim}$. Since $\sim \leq \approx$, we have $A^{\sim} \subseteq A^{\approx}$. It therefore suffices to prove that, if $\langle u, v\rangle \in A^{\approx}$, there exists a path of $A^{\sim}$ joining $u$ to $v$. Suppose $\langle u, v\rangle \in A \approx$, so that $C_{u} \approx C_{v}$. By the definition of $\approx$, there exist vertices $u_{0}=u, u_{1}, \ldots, u_{k}=v$ such that, for $0 \leq i<k, C_{u_{i}}$ and $C_{u_{i+1}}$ contain two $\sim$-equivalent rays. Therefore, $\left\langle u_{i}, u_{i+1}\right\rangle \in A^{\sim}$, and hence $u$ and $v$ are connected by a path in $A^{\sim}$. The proof is complete.

Proof of Theorem 6.3. This is inspired by the proof of [8, Thm 5.3(c)]; see also [18, Thm 1.3]. For any cutset $C$, we write ins $(C)$ for the set of all vertices reachable from 0 along paths of $T$ disjoint from $C$, and $\bar{C}=C \cup \operatorname{ins}(C)$. The sub- $\sigma$-field of $\mathcal{F}$ generated by the states of edges having no more than one vertex in $\bar{C}$ is denoted $\mathcal{T}_{C}$.

Let $\pi \geq p_{\mathrm{b}}(m)$ and $\sim \in \mathcal{E}_{\mathrm{o}}$. Choose $k$ according to Theorem 5.5(c), and let $W$ be the set of all vertices at distance $k$ from 0 . Let $A$ be an increasing cylinder event in $\mathcal{F}$, and 
suppose that $A$ is defined on the set of edges in some box $B$ of the tree which we may take sufficiently large to contain $W$. Let $\phi \in \mathcal{R}_{p, q}^{\sim}$. We shall show that

$$
\phi(A)=\phi_{p, q}^{1, \sim}(A),
$$

and the claim will follow.

Let $\Lambda$ be a box satisfying $\Lambda \supseteq B$ and let $\omega \in \Omega$. Recall from Section 2 that a vertex $x$ is blue if there exists an $x$-ray that is open (for the configuration $\omega$ ). For any box $\Delta \supseteq \Lambda$, let $\mathcal{B}_{\Delta}=\mathcal{B}_{\Delta}(\omega)$ be the set of blue cutsets contained in $\Delta$, noting that $\mathcal{B}_{\Delta}=\emptyset$ is possible. There is a natural partial order on $\mathcal{B}_{\Delta}$ given by $C_{1} \leq C_{2}$ if $C_{1} \subseteq \bar{C}_{2}$. Let $C_{\max }$ be the maximal blue cutset in this partial ordering, thus,

$$
\overline{C_{\max }}=\bigcup_{C \in \mathcal{B}_{\Delta}} \bar{C} .
$$

Note that, for any cutset $C \subseteq \Delta$, the event $E_{\Delta, C}=\left\{C_{\max }=C\right\}$ lies in the $\sigma$-field $\mathcal{T}_{C}$.

Let $\epsilon>0$. There exists a deterministic box $\Delta^{\prime}=\Delta^{\prime}(\Lambda, \epsilon) \supseteq \Lambda$ such that

$$
\phi_{\pi}\left(E_{\Delta}\right)>1-\epsilon \text { for all } \Delta \supseteq \Delta^{\prime},
$$

where $E_{\Delta}$ is the event

$$
E_{\Delta}=\left\{\mathcal{B}_{\Delta} \neq \emptyset \text { and } \Lambda \subseteq \operatorname{ins}\left(C_{\max }\right)\right\}=\bigcup_{\operatorname{ins}(C) \supseteq \Lambda} E_{\Delta, C} .
$$

Corollary 2.3 and the assumption $\pi \geq p_{\mathrm{b}}(m)$ have been used here. The corollary was phrased for the tree $T_{m}$ rather than $T_{m}^{\prime}$, but it is easily seen to be valid for either tree.

Let $C$ be a cutset with $\Lambda \subseteq \operatorname{ins}(C), C \subseteq \Delta$. On the event $E_{\Delta, C}, C$ is a blue cutset. By (4.6) and the fact that $W \subseteq B \subseteq \operatorname{ins}(C)$,

$$
\phi\left(A \mid \mathcal{T}_{C}\right)=\phi_{\bar{C}, p, q}^{1, \sim}(A) \quad \phi \text {-a.s., on } E_{\Delta, C} .
$$

By the FKG inequality (see (4.5),

$$
\phi_{\Delta, p, q}^{1, \sim}(A) \leq \phi\left(A \mid \mathcal{T}_{C}\right) \leq \phi_{\Lambda, p, q}^{1, \sim}(A) \quad \phi \text {-a.s., on } E_{\Delta, C} .
$$

We take expectations and sum over cutsets $C \subseteq \Delta$ such that $\Lambda \subseteq \operatorname{ins}(C)$ to find that

$$
\phi_{\Delta, p, q}^{1, \sim}(A) \phi\left(E_{\Delta}\right) \leq \phi(A)-\phi\left(A, \text { not } E_{\Delta}\right) \leq \phi_{\Lambda, p, q}^{1, \sim}(A) \phi\left(E_{\Delta}\right) .
$$

By (6.13) and the fact from Theorem 6.1 (c) that $\phi \geq_{\mathrm{st}} \phi_{\pi}$, if $\Delta$ is large enough,

$$
0 \leq \phi\left(A, \text { not } E_{\Delta}\right) \leq 1-\phi\left(E_{\Delta}\right) \leq 1-\phi_{\pi}\left(E_{\Delta}\right)<\epsilon .
$$

We pass to the limits in (6.14) as $\Delta \uparrow V, \epsilon \downarrow 0$, and $\Lambda \uparrow V$ to obtain (6.12) as required.

Proof of Theorem 6.4 Let $|x|$ denote the length of the unique path of $T_{m}^{\prime}$ from 0 to $x$. Fix $k \geq 0$, let $\Lambda_{k}$ be the set of all $x$ with $|x| \leq k$, and let $\partial \Lambda_{k}=\Lambda_{k} \backslash \Lambda_{k-1}$ as usual. We call 
$y$ a descendant of $x$ if the unique path from 0 to $y$ passes through $x$. For $x \in V$, write $D_{m}(x)$ for the set of all descendants $y$ of $x$ with $|y|=m$, and $D(x)=\bigcup_{m>|x|} D_{m}(x)$.

Choose $\sim \in \mathcal{E}_{\mathrm{o}}$, and fix $k=k(\sim)$ such that the conclusion of Theorem 5.5 (c) holds. Let $\sim_{k}$ be given as in that theorem. For $x, y \in \partial \Lambda_{k}$, we write $x \sim_{k} y$ if the unique paths $\pi_{0, x}$ (respectively, $\pi_{0, y}$ ) from 0 to $x$ (respectively, $y$ ) satisfy $\pi_{0, x} \sim_{k} \pi_{0, y}$. We have

$$
\phi_{p, q}^{1, \sim}=\lim _{n \rightarrow \infty} \psi_{n}^{\sim},
$$

where $\psi_{n}^{\sim}=\phi_{\Lambda_{n}, p, q}^{1, \sim}$. Let $n>k=k(\sim)$. The measure $\psi_{n}^{\sim}$ may be considered as the random-cluster measure on the graph $G_{n}^{\sim}=\left(\Lambda_{n}^{\sim}, E_{\Lambda_{n}}\right)$, where $\Lambda_{n}^{\sim}$ is obtained from $\Lambda_{n}$ by identifying any pair of vertices $u, v \in \partial \Lambda_{n}$ having the property that there exist $\rho_{u} \in \mathcal{R}_{u}, \rho_{v} \in \mathcal{R}_{v}$ with $\rho_{u} \sim \rho_{v}$. Since $n>k$, this implies two levels of identifications:

(i) for every $x \in \partial \Lambda_{k}$, we identify the set $D_{n}(x)$ of vertices,

(ii) if $x, y \in \partial \Lambda_{k}$ are such that $x \sim_{k} y$, we identify all vertices in $D_{n}(x) \cup D_{n}(y)$.

Part (ii) incorporates part (i), since $x \sim_{k} x$ for $x \in \partial \Lambda_{k}$, but we express it thus in order to emphasize the role of the equivalence relation $\sim$.

We recall a basic fact (see [11], especially the appendix). Consider the random-cluster measure on a finite graph $G=(A, B)$ with cluster-weighting factor $q$ and a family $\mathbf{p}$ $=\left(p_{b}: b \in B\right)$ of edge-parameters. Any set $B^{\prime}$ of one or more edges in parallel (respectively, in series) may be replaced by a single edge with an associated edge-parameter that is a function of $\left(p_{b}: b \in B^{\prime}\right)$ and $q$. We call such a replacement a parallel (respectively, series) replacement.

From the finite subtree $\left(\Lambda_{k}, E_{\Lambda_{k}}\right)$ of $T_{m}^{\prime}$ we construct as follows a further tree denoted $T$. To each $x \in \partial \Lambda_{k}$ we attach a further edge $\left[x, x^{\prime}\right\rangle$ to the vertex $x$, arriving thus at a tree which we denote $T$ and which is illustrated in Figure 6.1 These new edges are called attachments. From $T$ we obtain the graph $T^{\sim}$ by identifying $x^{\prime}$ and $y^{\prime}$ whenever $x \sim_{k} y$. The graph $G_{n}^{\sim}$ may be transformed by a sequence of parallel and series replacements into $T^{\sim}$. The random-cluster measure $\psi_{n}^{\sim}$ corresponds to the random-cluster measure $v_{n}^{\sim}$ on $T^{\sim}$ for which the attachment edge $\left[x, x^{\prime}\right\rangle$ has an associated parameter $p_{n}$ that depends only on $p, q, k$, and $n$, and not further on the choice of $x$ and $\sim$. Since

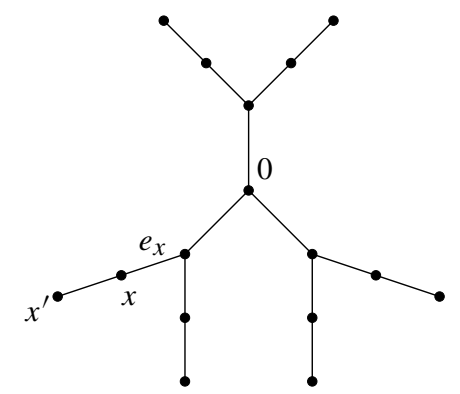

Fig. 6.1. To each boundary vertex $x$ of the first $k(=2)$ generations of $T_{m}^{\prime}$ is attached a new edge $\left[x, x^{\prime}\right\rangle$. 
$\psi_{n}^{\sim} \Rightarrow \phi_{p, q}^{1, \sim}$, we see that $p_{n} \rightarrow p_{\infty}$ for some $p_{\infty} \in[0,1]$, and that $\phi_{p, q}^{1, \sim}$ corresponds to the random-cluster measure $\nu_{\infty}^{\sim}$ on $T^{\sim}$ for which the attachment edges have associated parameter $p_{\infty}$.

Assume now that $p$ is such that $0<\theta^{1}(p, q)<1$. It is easily deduced that $p_{\infty} \in$ $(0,1)$. For $x \in \partial \Lambda_{k}$, we write $e_{x}$ for the unique edge of $T_{m}^{\prime}$ of the form $[z, x\rangle$. The marginal (joint) law of the states of the edges $\left(e_{x}: x \in \partial \Lambda_{k}\right)$ is the same under $\phi_{p, q}^{1, \sim}$ as under $v_{\infty}^{\sim}$.

Let $q>1$, and let $x, y \in \partial \Lambda_{k}, x \neq y$. Let $C$ be the event that $e_{z}$ is closed for all $z \in \partial \Lambda_{k} \backslash\{x, y\}$. The conditional measure given $C$ of $\nu_{\infty}^{\sim}$ is a random-cluster measure on the graph $T^{\sim}$ with the edges $e_{z}, z \in \partial \Lambda_{k} \backslash\{x, y\}$, removed, and we denote this graph by $T_{x, y}^{\sim}$. It is a fundamental property of random-cluster measures with $q \neq 1$ on a finite graph, with edge-parameters lying in $(0,1)$, that the states of two edges are dependent random variables if and only if there exists some cycle containing both edges (see [11]). There exists a cycle in $T_{x, y}^{\sim}$ containing both $e_{x}$ and $e_{y}$ if and only if $x \sim_{k} y$. Therefore, $x \sim_{k} y$ if and only if

$$
\nu_{\infty}^{\sim}\left(J_{x} \mid J_{y} \cap C\right) \neq \tilde{\nu}_{\infty}^{\sim}\left(J_{x} \mid J_{y}^{\mathrm{c}} \cap C\right),
$$

where $J_{x}=J_{e_{x}}$ is the event that $e_{x}$ is open.

The proof is nearly complete. Let $\sim$ and $\sim^{\prime}$ be distinct open equivalence relations, and choose $k$ sufficiently large that the conclusion of Theorem 5.5. (c) holds for both and $\sim^{\prime}$. Since $\sim$ and $\sim^{\prime}$ are distinct, there exist $x, y \in \partial \Lambda_{k}$ such that $x \sim_{k} y$ but $x \nsim_{k}^{\prime} y$. Therefore, $v_{\infty}^{\sim}$ satisfies (6.15) but $v_{\infty}^{\sim^{\prime}}$ does not, and hence $\phi_{p, q}^{1, \sim} \neq \phi_{p, q}^{1, \sim^{\prime}}$ as required.

Acknowledgements. This work was done during a visit by the second author to Cambridge University with support from the Royal Swedish Academy of Sciences, the London Mathematical Society, and Churchill College, Cambridge.

\section{References}

[1] Athreya, K. B., Ney, P. E.: Branching Processes. Springer, New York (1972) Zbl 0259.60002 MR 0373040

[2] Bleher, P. M., Ruiz, J., Zagrebnov, V. A.: On the purity of the limiting Gibbs state for the Ising model on the Bethe lattice. J. Statist. Phys. 79, 473-482 (1995) Zbl pre01553929 MR 1325591

[3] Bleher, P. M., Ruiz, J., Schonmann, R. H., Shlosman, S., Zagrebnov, V. A.: Rigidity of the critical phases on a Cayley tree. Moscow Math. J. 1, 345-363, 470 (2001) Zbl 0998.60022 MR 1877597

[4] Bollobás, B.: Random Graphs. Academic Press, London (1985) Zbl 0567.05042 MR 0809996

[5] Bollobás, B., Grimmett, G. R., Janson, S.: The random-cluster process on the complete graph. Probab. Theory Related Fields 104, 283-317 (1996)

[6] Cohn, D. L.: Measure Theory. Birkhäuser, Boston (1980) Zbl 0436.28001 MR 0578344

[7] Georgii, H.-O., Häggström, O., Maes, C.: The random geometry of equilibrium phases. In: Phase Transitions and Critical Phenomena Vol. 18, C. Domb and J. L. Lebowitz (eds.), Academic Press, London, 1-142 (2000) MR 2014387 
[8] Grimmett, G. R.: The stochastic random-cluster process and the uniqueness of random-cluster measures. Ann. Probab. 23, 1461-1510 (1995) Zbl 0852.60105 MR 1379156

[9] Grimmett, G. R.: Percolation. 2nd ed., Springer, Berlin (1999) Zbl 0926.60004 MR 1707339

[10] Grimmett, G. R.: The random-cluster model. In: Probability on Discrete Structures, H. Kesten (ed.), Encyclopaedia Math. Sci. 110, Springer, Berlin, 73-123 (2003) Zbl 1045.60105 MR 2023651

[11] Grimmett, G. R.: The Random-Cluster Model. In preparation (2004)

[12] Grimmett, G. R., Stirzaker, D. R.: Probability and Random Processes. 3rd ed., Oxford Univ. Press, Oxford (2001) Zbl 1015.60002 MR 2059709

[13] Häggström, O.: The random-cluster model on a homogeneous tree. Probab. Theory Related Fields 104, 231-253 (1996) Zbl 0838.60086 MR 1373377

[14] Häggström, O., Jonasson, J., Lyons, R.: Explicit isoperimetric constants and phase transitions in the random-cluster model. Ann. Probab. 30, 443-473 (2002) Zbl 1025.60044 MR 1894115

[15] Harris, T. E.: The Theory of Branching Processes. Dover, Mineola, NY (1989) (first published in 1963) Zbl 1037.60001 MR 1991122

[16] Jagers, P.: Branching Processes with Biological Applications. Wiley, Chichester (1975) Zbl 0356.60039 MR 0488341

[17] Janson, S., Łuczak, T., Ruciński, A.: Random Graphs. Wiley, New York (2000) Zbl 0968.05003 MR 1782847

[18] Jonasson, J.: The random cluster model on a general graph and a phase transition characterization of nonamenability. Stochastic Process. Appl. 79, 335-354 (1999) Zbl 0963.60095 MR 1671859

[19] Liggett, T. M.: Interacting Particle Systems. Springer, Berlin (1985) Zbl 0559.60078 MR 0776231

[20] Luczak, M., Łuczak, T.: The phase transition in the cluster-scaled model of a random graph. To appear (2004)

[21] Martin, J.: Reconstruction thresholds on regular trees. Discrete Math. Theor. Comput. Sci., Proc. AC, Nancy, 191-204 (2003) Zbl pre02046068 MR 2042387

[22] Mossel, E.: Survey: Information flow on trees. In: Graphs, Morphisms and Statistical Physics, J. Nešetřil and P. Winkler (eds.), DIMACS Ser. Discrete Math. Theoret. Comput. Sci. 63, Amer. Math. Soc., 155-170 (2004) MR 2056226 